ARTICLE

\title{
AGPAT2 interaction with CDP-diacylglycerol synthases promotes the flux of fatty acids through the CDP-diacylglycerol pathway
}

Hoi Yin Mak (1) 1,11, Qian Ouyang 2,11, Sergey Tumanov 3,4,5,11, Jiesi Xu6 ${ }^{6}$, Ping Rong², Feitong Dong7 , Sin Man Lam (10 6,8, Xiaowei Wang9, Ivan Lukmantara1, Ximing Du (1) 1, Mingming Gao9 , Andrew J. Brown?',

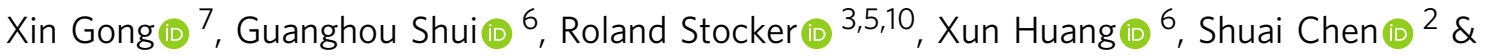
Hongyuan Yang (iD ${ }^{1 凶}$

AGPATs (1-acylglycerol-3-phosphate O-acyltransferases) catalyze the acylation of lysophosphatidic acid to form phosphatidic acid (PA), a key step in the glycerol-3-phosphate pathway for the synthesis of phospholipids and triacylglycerols. AGPAT2 is the only AGPAT isoform whose loss-of-function mutations cause a severe form of human congenital generalized lipodystrophy. Paradoxically, AGPAT2 deficiency is known to dramatically increase the level of its product, PA. Here, we find that AGPAT2 deficiency impairs the biogenesis and growth of lipid droplets. We show that AGPAT2 deficiency compromises the stability of $\underline{C D P}$ diacylglycerol (DAG) synthases (CDSs) and decreases CDS activity in both cell lines and mouse liver. Moreover, AGPAT2 and CDS1/2 can directly interact and form functional complexes, which promote the metabolism of PA along the CDP-DAG pathway of phospholipid synthesis. Our results provide key insights into the regulation of metabolic flux during lipid synthesis and suggest substrate channelling at a major branch point of the glycerol-3-phosphate pathway.

\footnotetext{
${ }^{1}$ School of Biotechnology and Biomolecular Sciences, the University of New South Wales, Sydney, NSW 2052, Australia. ${ }^{2}$ MOE Key Laboratory of Model Animal for Disease Study, Model Animal Research Center, School of Medicine, Nanjing University, 210061 Nanjing, China. ${ }^{3}$ Heart Research Institute, The University of Sydney, Newtown, NSW 2042, Australia. ${ }^{4}$ Faculty of Medicine and Health, The University of Sydney, Sydney, NSW 2006, Australia. ${ }^{5}$ Victor Chang Cardiac Research Institute, Darlinghurst, NSW 2010, Australia. ${ }^{6}$ State Key Laboratory of Molecular Developmental Biology, Institute of Genetics and Developmental Biology, Chinese Academy of Sciences, 100101 Beijing, China. ${ }^{7}$ Department of Biology, Southern University of Science and Technology, 518055 Shenzhen, Guangdong, China. ${ }^{8}$ Lipidall Technologies Company Limited, 213022 Changzhou, Jiangsu Province, China. ${ }^{9}$ Laboratory of Lipid Metabolism, Hebei Medical University, 050017 Shijiazhuang, Hebei, China. ${ }^{10}$ School of Life and Environmental Sciences, The University of Sydney, Sydney, NSW 2006, Australia. ${ }^{11}$ These authors contributed equally: Hoi Yin Mak, Qian Ouyang, Sergey Tumanov. ${ }^{凶}$ email: h.rob.yang@unsw.edu.au
} 
iving organisms need to store energy to survive in an everchanging environment. For cells, energy is stored in the form of neutral lipids within lipid droplets (LDs), which are evolutionarily conserved organelles found in nearly all organisms ${ }^{1-3}$. Mammals have developed adipocytes and adipose tissue, which specialize in energy storage ${ }^{4,5}$. Adipocytes are derived from mesenchymal stem cells through a process called adipogenesis that is regulated by a transcriptional cascade. Understanding the fundamental mechanisms governing LD formation/growth (cellular lipid storage) and adipogenesis (systemic lipid storage) may provide better treatment strategies against obesity and its associated metabolic disorders.

The 1-acylglycerol-3-phosphate $O$-acyltransferases (AGPATs), also known as lysophosphatidic acid acyltransferases (LPAATs) are intermediate enzymes in the biosynthesis of phospholipids and triacylglycerols (TAGs) through the glycerol-3-phosphate pathway (Fig. 1a) ${ }^{6,7}$. The first committed step is the acylation of glycerol-3-phosphate to form 1-acylglycerol-3-phosphate (also called lysophosphatidic acid (LPA)). This reaction is catalyzed by glycerol-3-phosphate $O$-acyltransferases (GPATs). AGPATs/ LPAATs esterify the $s n-2$ position of LPA to make phosphatidic acid (PA), a critical intermediate that can be dephosphorylated into diacylglycerol (DAG) by PA phosphatases (PAPs, e.g., lipins), or converted into CDP-DAG by CDP-DAG synthases 1 and $2(\operatorname{CDS} 1 \text { and } 2)^{8}$. To date, at least 5 putative human AGPAT isoforms have been identified, each encoded by a different gene ${ }^{7}$. Among the AGPAT isoforms, AGPAT2 stands out since null mutations of AGPAT2 are associated with Berardinelli-Seip congenital lipodystrophy type 1 (BSCL1)/congenital generalized lipodystrophy, type 1 (CGL1), which is characterized by a near complete loss of adipose tissue, early onset of insulin resistance, diabetes, hypertriglyceridemia, and hepatic steatosis ${ }^{9}$. Human AGPAT2 localizes to the endoplasmic reticulum (ER), and is expressed abundantly in adipose tissue, liver and pancreas ${ }^{10}$. Importantly, Agpat $2^{-/}$mice replicate most of the features of human BSCL1/CGL1, although the extent of fat loss and the degree of insulin resistance appear more severe in mice than in humans ${ }^{11}$.

Exactly how AGPAT2 deficiency causes lipodystrophy, i.e., BSCL1, is unknown ${ }^{11,12}$. While AGPAT2 catalyzes the acylation of LPA to PA, the level of PA was surprisingly and dramatically increased in AGPAT2-deficient cells and liver ${ }^{11-13}$. In this connection, seipin, whose loss-of-function mutations cause BSCL2, also regulates the level and distribution of $\mathrm{PA}^{14-18}$. PA and cyclic PA have been shown to antagonize PPAR $\gamma$, a ligand-activated transcription factor essential for adipogenesis ${ }^{19,20}$. Thus, both AGPAT2 and seipin could regulate adipogenesis through modulating the level/distribution of PA. Moreover, PA is the only cone-shaped anionic lipid in a cell ${ }^{21}$. Due to its shape and negative charge, PA's concentration/distribution can greatly impact membrane function. An increase in local PA content may affect ER surface tension/curvature and alter the biogenesis/ growth of $\mathrm{LDs}^{15,17,22}$. Indeed, seipin has been established as a key regulator of the initiation and growth of $\mathrm{LDs}^{14,22-24}$. If and how AGPAT2 may impact LD formation remains unexplored.

In this work, we set out to investigate LD initiation and growth in AGPAT2-deficient cells. We discover an unexpected link between AGPAT2 and CDS1/2, enzymes that convert PA to CDP-DAG for the synthesis of phospholipids including phosphatidylinositol (PI) and phosphatidylglycerol (PG) (Fig. 1a). Our results unveil a mechanism by which AGPAT2 deficiency may lead to an increase of cellular PA and cause lipodystrophy. More broadly, our results suggest the existence of substrate channelling at a key branch point of the glycerol-3-phosphate pathway for the synthesis of phospholipids and TAGs.

\section{Results}

AGPAT2 deficiency results in the formation of supersized LDs and a delay in initial LD lipidation. Tagged and overexpressed human AGPAT2 was shown to localize to the $\mathrm{ER}^{10}$. However, the localization of endogenous AGPAT2 has not been determined, possibly due to a lack of an appropriate anti-AGPAT2 antibody. We tagged AGPAT2 with super folder GFP (sfGFP) at its genomic locus by CRISPR, and found that AGPAT2-sfGFP colocalized with calnexin, an ER marker (Supplementary Fig. 1a). Interestingly, a portion of AGPAT2 appeared very close to LDs (Supplementary Fig. 1a). The fluorescence signal disappeared after knocking-down AGPAT2 by siRNA, indicating the accuracy of GFP integration (Supplementary Fig. 1a, b).

Given its ER localization and known impact on PA metabolism, AGPAT2 may regulate the formation of LDs. To test this hypothesis, AGPAT2 was knocked down by siRNA in three different cells lines: HeLa, Huh7 and AML12. AGPAT2 deficiency led to the formation of giant LDs (defined as LDs with diameters $>2 \mu \mathrm{m})$ after prolonged oleate treatment $(18 \mathrm{~h})$ in all three cell lines (Fig. 1b-h). The amount of TAG was also significantly increased under AGPAT2 deficiency (Supplementary Fig. 1c). We also knocked out AGPAT2 in these cells by CRISPR, but the knock-out cells appeared very sick (data not shown) and therefore not used. Wild type (WT) AGPAT2, but not a catalytic inactive mutant $(\mathrm{H} 98 \mathrm{~A})^{25}$, rescued the LD phenotype when expressed in the knockdown cells (Fig. 1i, j). Moreover, the sizes of LDs were also increased when AGPAT2 was knocked down in 3T3 L1 adipocytes, a professional cell for fat storage (Supplementary Fig. 1d-f). Knocking down AGPAT2 did not seem to impact the viability of 3T3 L1 adipocytes (Supplementary Fig. 1g). To characterize the formation of supersized LDs in greater detail, we examined $\mathrm{LD}$ formation over $24 \mathrm{~h}$ of oleate treatment. Within $2 \mathrm{~h}$ of oleate addition, LDs in AGPAT2-depleted cells were larger than those in control cells, although almost all LDs were $<2 \mu \mathrm{m}$ in diameter (Supplementary Fig. 1h, i). Giant LDs first appeared in AGPAT2 knockdown cells $\sim 8 \mathrm{~h}$ after adding oleate and became more prominent later. In contrast, giant LDs were extremely rare in control cells.

LDs are believed to originate from the ER via several steps. First, TAGs are synthesized within the two leaflets of the ER by diacylglycerol acyltransferases (DGATs) ${ }^{26,27}$. When newly synthesized TAG reaches a critical mass, it is thought to nucleate and bud from discrete sites of the ER as initial LDs. This nucleation and budding process may be controlled by both proteins such as seipin and/or ER membrane phospholipids and surface tension $22,23,28$. Given that PA, a negatively charged conical lipid, was dramatically increased in AGPAT2-deficient cells $^{11-13}$, we wondered if AGPAT2 deficiency may also impact the early steps of LD formation. To investigate this possibility, we tagged endogenous perilipin 3 (PLIN3) by genome engineering with mCherry as described ${ }^{23}$. PLIN3 is an endogenous protein that indicates the earliest steps of LD formation whereas lipophilic dyes such as LipidTox/BODIPY only stain LDs that have acquired substantial neutral lipids. We also used seipin knockout cells as a control because seipin deficiency is known to impair early LD formation ${ }^{23,29}$. In WT cells, when LD formation was induced by oleate addition, PLIN3 rapidly accumulated in small puncta that grew larger, and became BODIPY-positive within $20 \mathrm{~min}$ (Fig. 2a, b). In both seipin- and AGPAT2-deficient cells, there was a sharp increase in the number of PLIN3 puncta within $10 \mathrm{~min}$ of oleate treatment (Fig. 2a, b; Supplementary Fig. 2a). Moreover, there were much fewer BODIPY-positive LDs in AGPAT2-deficient cells within 10 min of oleate treatment than in WT cells (Fig. 2a, b; Supplementary Fig. 2b). In seipin-deficient cells, the delay in forming BODIPY-positive LDs is more 

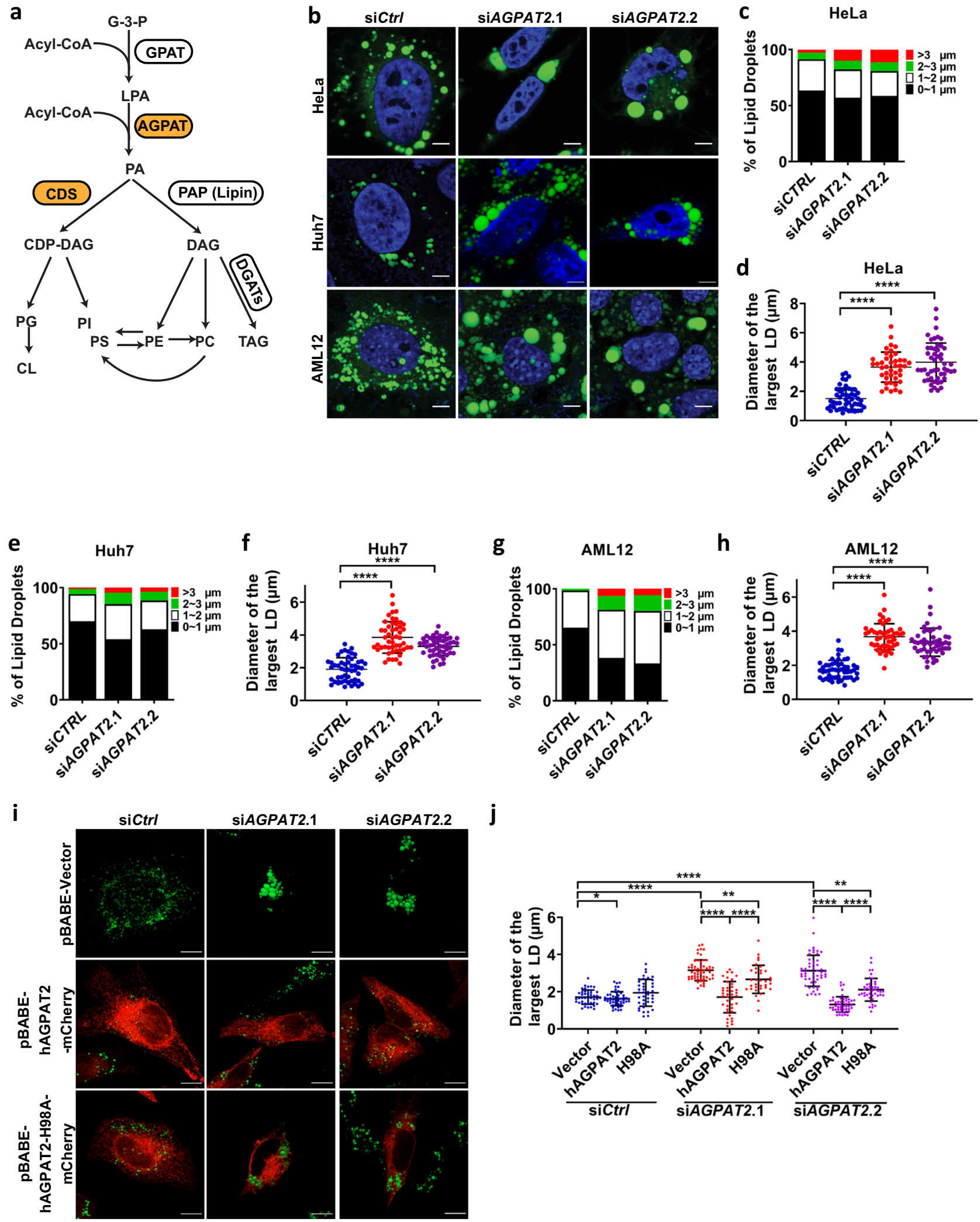

pronounced as few PLIN3 puncta became BODIPY-positive after 18 min of oleate treatment (Fig. 2a, b; Supplementary Fig. 2b).

Reducing PA restores normal LD morphology in AGPAT2deficient cells. We next aimed to determine the molecular basis for the aberrant LD biogenesis and formation under AGPAT2 deficiency. Knocking down DGAT1 or 2 with established siRNAs did not completely restore the normal size of LDs in AGPAT2deficient cells (Fig. 3a, b) ${ }^{30}$. This suggests that mechanisms other than TAG synthesis may also be responsible for increased LD size. As mentioned above, an increase in whole cell PA in AGPAT2-deficient cells has been reported by different groups $^{11-13}$. This was further confirmed here by using a PA 
Fig. 1 AGPAT2 depletion alters LD morphology. a The glycerol-3-phosphate (G-3-P) pathway for the synthesis of phospholipids and triacylglycerols. GPAT glycerol-3-phosphate O-acyltransferase, AGPAT 1-acylglycerol-3-phosphate O-acyltransferase, LPA lysophosphatidic acid, PA phosphatidic acid, PAP PA phosphatase, CDS CDP-DAG synthase, DAG diacylglycerol, PS phosphatidylserine, PE phosphatidylethanolamine, PC phosphatidylcholine, TAG triacylglycerol, PG phosphatidylglycerol, PI phosphatidylinositol, CL cardiolipin. b Confocal imaging of LDs in control and HeLa, Huh7 and AML12 cells treated with AGPAT2 siRNA. The cells were treated with $20 \mathrm{nM}$ siRNA for $24 \mathrm{~h}$, followed by oleate treatment $(400 \mu \mathrm{M})$ for $18 \mathrm{~h}$. Blue represents DAPI staining, and green represents BODIPY staining. Bars: $5 \mu \mathrm{m}$. c, e, $\mathbf{g}$ Bar graphs show LD size distribution in HeLa, Huh7 and AML12 cells. Diameter of all detectable LDs in a cell was measured and represented by red $(>3 \mu \mathrm{m})$, green $(2-3 \mu \mathrm{m})$, white $(1-2 \mu \mathrm{m})$ and black $(0-1 \mu \mathrm{m})(n=42-50$ cells examined over 3 biologically independent experiments). $\mathbf{d}, \mathbf{f}, \mathbf{h}$ Quantification of LD diameters in HeLa, Huh7 and AML12 cells. Diameter of the largest LD in a cell was measured (mean $\pm \mathrm{SD}$; ${ }^{\star \star \star \star} p<0.0001$, one-way ANOVA, $n=42-50$ cells examined over three biologically independent experiments). i Human AGPAT2mCherry or the catalytic dead mutant H98A was overexpressed in AGPAT2 knockdown HeLa cells. Cells were treated with $400 \mu \mathrm{M}$ oleic acid for $18 \mathrm{~h}$. Green represents BODIPY staining and red indicates mCherry expression. Bars $=5 \mu \mathrm{m}$. $\mathbf{j}$ Quantification of LD diameters after overexpressing vector, AGPAT2-mCherry, and AGPAT2-H98A-mCherry in AGPAT2 knockdown HeLa cells. Diameter of the largest LD in a cell was measured (mean \pm SD; ${ }^{\star \star \star \star} p<0.0001 ;{ }^{\star \star} p<0.01$, two-way ANOVA, $n=31-59$ cells examined over three biologically independent experiments).

sensor (GFP-PDE4A1) in AGPAT2 knockdown HeLa cells ${ }^{31}$ : total fluorescence intensity of GFP-PDE4A1 in AGPAT2deficient cells was higher than that in control cells, and GFPPDE4A1 colocalized with calnexin, suggesting increased PA in the ER (Supplementary Fig. 3a, b). Among other possibilities, this increase in PA may underpin the formation of large LDs in AGPAT2-deficient cells. To examine this possibility, we overexpressed seipin, CDS1 and CDS2. Overexpressing any one of these three genes reduced cellular PA (Supplementary Fig. 3c, d) and almost completely abolished the formation of supersized LDs in AGPAT2-deficient cells (Fig. 3c-e). Together, these results suggest that increased PA is at least partially responsible for the abnormal LD formation in AGPAT2-deficient cells.

Reduced CDS protein expression and activity in AGPAT2deficient cells. While examining the effect of CDS1/CDS2 expression on LD formation in AGPAT2-deficient cells, we noticed that the fluorescence intensity of CDS1/2, but not seipin, was much weaker in AGPAT2-deficient than WT HeLa cells (red fluorescence, Fig. 3c). We further verified this observation in Huh7 cells (Fig. 4a and Supplementary Fig. 4a). The loss of mCherry-CDS1/2 signal in AGPAT2-deficient cells suggests a possible reduction of CDS1/2 protein mass and activity. This could explain the increased PA in AGPAT2-deficient cells because CDS1/CDS2-deficiency is known to result in the accumulation of PA and giant $\mathrm{LDs}^{15,30,32}$. We therefore examined the amount of CDS1 and CDS2 in WT and AGPAT2-deficient cells. Consistent with the imaging results, both HA-CDS1 and -CDS2 were reduced in AGPAT2-deficient cells (Fig. 4b-d). Moreover, the amount of endogenous CDS2 protein was significantly decreased in AGPAT2-deficient Huh7 cells (Fig. 4e). We were not able to quantify endogenous CDS1 due to the lack of a suitable antibody. The reduction in CDS1 and CDS2 was not due to transcriptional regulation, since CDS1/2 mRNA did not decrease in AGPAT2-deficient cells (Supplementary Fig. 4b). Similarly, AGPAT2 mRNA expression was not affected in CDS1/2 deficient cells despite a minor reduction in AGPAT2 protein (Fig. 4e and Supplementary Fig. 4b). The half-life of CDS1/2 is about $4-8 \mathrm{~h}$ in WT cells (Supplementary Fig. 4c-e). Under AGPAT2 deficiency, the steady state level of CDS1/2 was dramatically reduced and the effect of cycloheximide (CHX) became less clear (Supplementary Fig. $4 \mathrm{c}-\mathrm{e}$ ). Consistent with the reduction in protein expression, CDS activity was decreased by $\sim 30 \%$ in AGPAT2-deficient cells relative to control cells (Fig. 4f). Moreover, overexpressing AGPAT2, CDS1 or CDS2 increased CDS activity (Fig. 4g and Supplementary Fig. 4f). Overexpressing AGPAT2 also caused a small but significant decrease of ${ }^{14} \mathrm{C}$ oleate incorporation into TAG (Fig. 4h and Supplementary Fig. 4g). Finally, since there are five isoforms of AGPAT, we wonder if the loss of CDS1/2 was AGPAT2 specific. Knocking down AGPAT2, but none of the other AGPATs, consistently decreased CDS1/2 (Supplementary Fig. 4h-k). Together, these results support a specific functional relationship between AGPAT2 and CDS1/2.

AGPAT2 and CDS1/2 physically interact and form stable complexes. One possible explanation for the loss of CDS1/2 under AGPAT2 deficiency is that AGPAT2 may form a complex with CDS1/2 and promote their stability/activity. The interaction between AGPAT2 and CDS1/2 was therefore investigated. Human CDS1 and CDS2 both co-immunoprecipitated with human AGPAT2 with similar affinity (Fig. 5a, Supplementary Fig. 5a). As a control, seipin interacted with GPAT4, but not with AGPAT2 under the same conditions (Supplementary Fig. 5b). To further examine the AGPAT2-CDS interaction, we also expressed Strep-tagged AGPAT2 and Flag-tagged CDS1 or CDS2 in HEK293F cells, followed by a two-step affinity purification by anti-Flag and anti-Strep. A large amount of AGPAT2 and CDS1/ 2 co-purified as assessed by Coomassie blue, suggesting a direct and stable interaction (Fig. 5b and Supplementary Fig. 5c). To verify the interaction between AGPAT2 and CDSs, we tagged AGPAT2 with sfGFP and CDS2 with mScarlet at their respective genomic locus by CRISPR (Supplementary Fig. 5d). For unknown reasons, we were not able to tag CDS1 despite multiple attempts. Notably, knocking down AGPAT2 not only reduced the level of AGPAT2-sfGFP, but also that of CDS2-mScarlet, further demonstrating that AGPAT2 is required for the stability of CDS2 as shown in Fig. 4 (Supplementary Fig. 5d). Importantly, AGPAT2-sfGFP and CDS2-mScarlet coprecipitated (Fig. 5c). There are two isoforms of AGPAT2 and CDS2 appears to interact stronger with the longer isoform (Fig. 5c and Supplementary Fig. 5d). As a further proof of the dynamic interaction between AGPAT2 and CDS2, there appears to be enhanced colocalization between endogenous AGPAT2 and CDS2 when cells were cultured in low glucose media (Fig. 5d and Supplementary Fig. 5e). The enhanced colocalization was reversed upon adding back glucose. We further determined if the apparent interaction between AGPAT2 and CDS1/2 is unique since there are five AGPAT isoforms. Co-immunoprecipitation (co-IP) experiments using AGPAT1-5 and CDS1/2 identified AGPAT2 as the major AGPAT isoform to co-precipitate with CDS1/CDS2 (Fig. 5e-h; Supplementary Fig. 5f-i), consistent with its specific functional link with $\mathrm{CDS} 1 / 2$.

AGPAT2 promotes the flux of oleate through the CDP-DAG pathway. PA generated by AGPATs is a key branch point metabolite in the synthesis of phospholipids and TAGs: PA can be used directly by CDS1/CDS2 for the synthesis of CDP-DAG or by PAPs (e.g., lipins) for the synthesis of DAG. Substrate channelling often occurs at metabolic branch points ${ }^{33,34}$. AGPAT2 


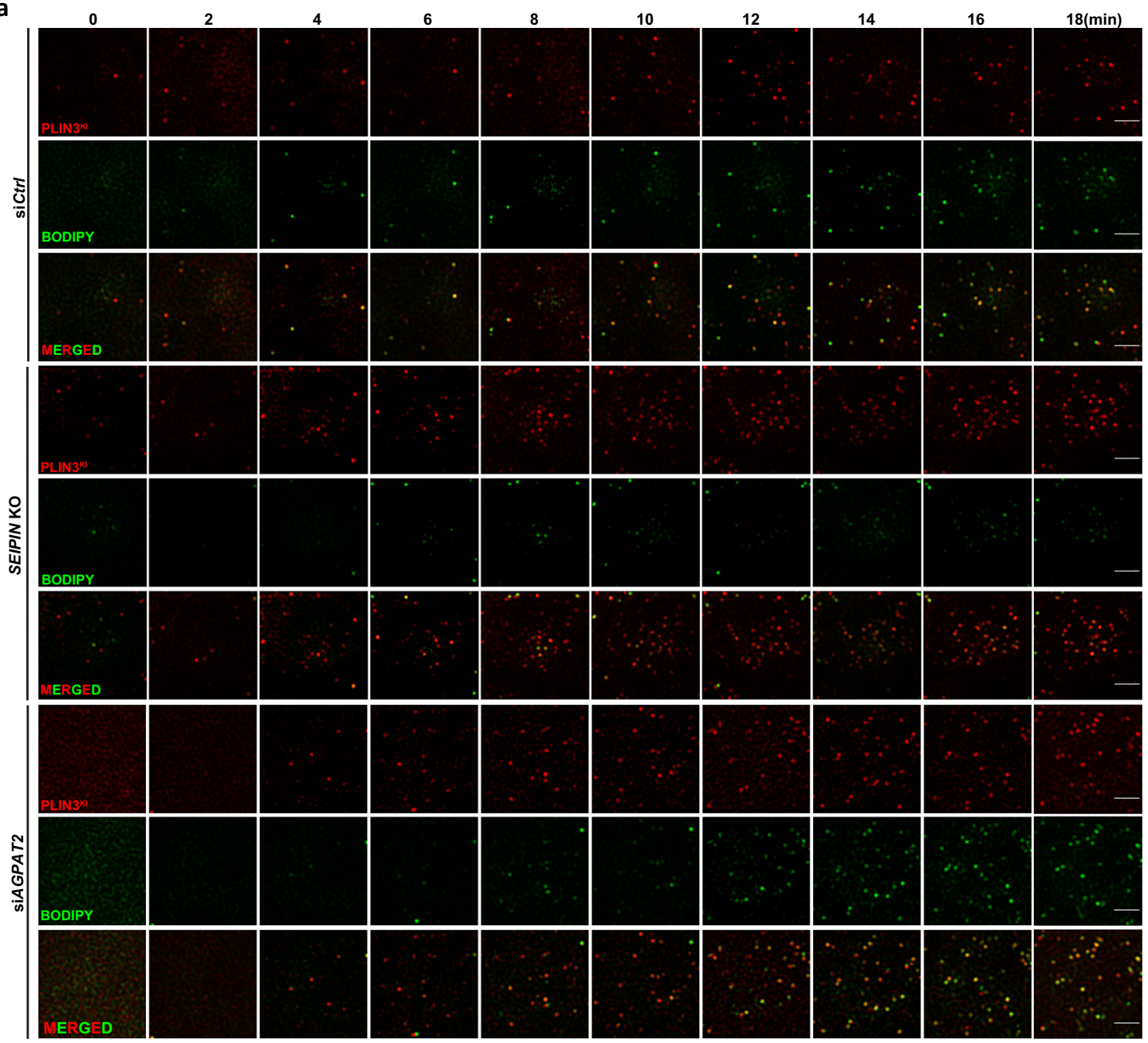

b
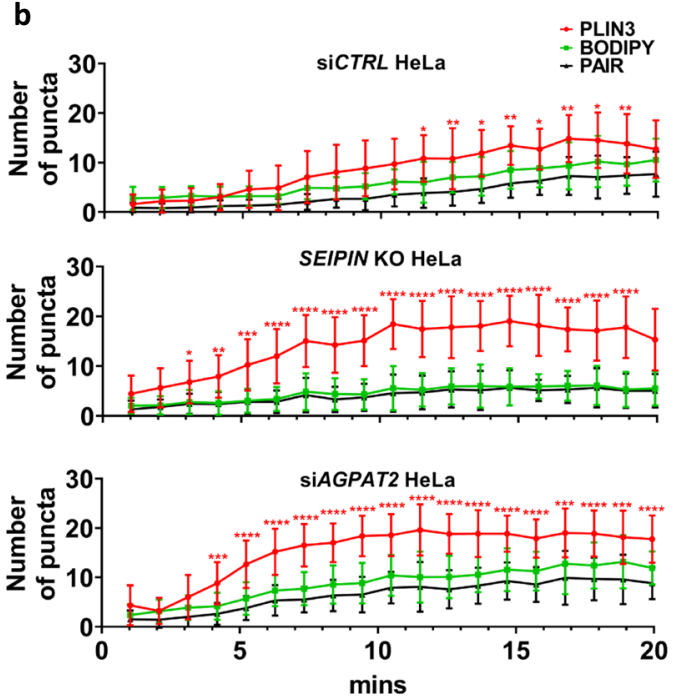

Fig. 2 AGPAT2 depletion impacted initial LD formation. a Control, seipin knockout (KO) and AGPAT2 knockdown HeLa cells deficient were starved in 1\% LPDS for $16 \mathrm{~h}$. Representative images show the localization pattern of endogenous PLIN3 (mCherry-tagged) and BODIPY puncta every two minutes after oleate addition $(400 \mu \mathrm{M})$. Bars: $5 \mu \mathrm{m}$. b The number of PLIN3 and BODIPY puncta in control, seipin KO and AGPAT2 knockdown HeLa cells at indicated time points. Pair indicates colocalization of PLIN3 and BODIPY puncta. (mean \pm SD; ${ }^{\star \star \star \star} p<0.0001 ;{ }^{\star \star \star} p<0.001 ;{ }^{\star \star} p<0.01 ;{ }^{\star} p<0.05$, two-way ANOVA, $n=15-20$ cells examined over three biologically independent experiments). 
a

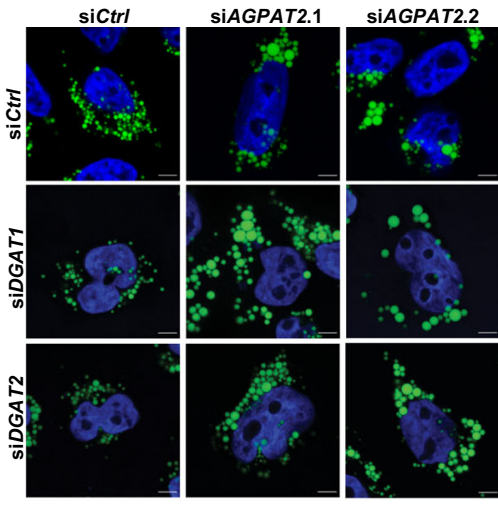

b

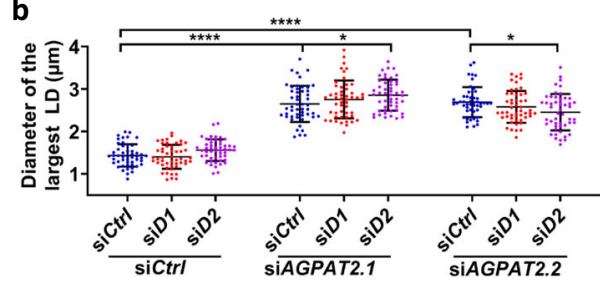

C
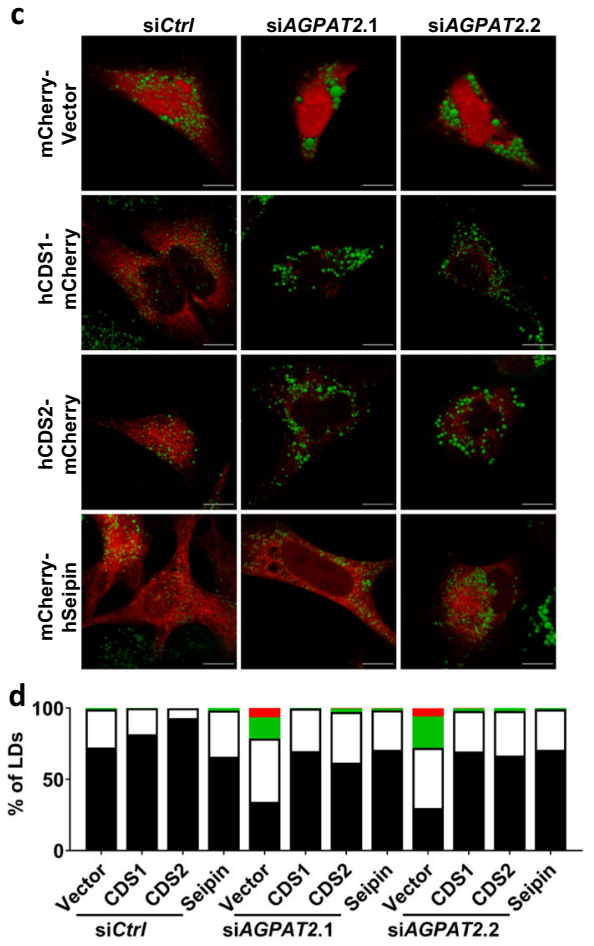

e

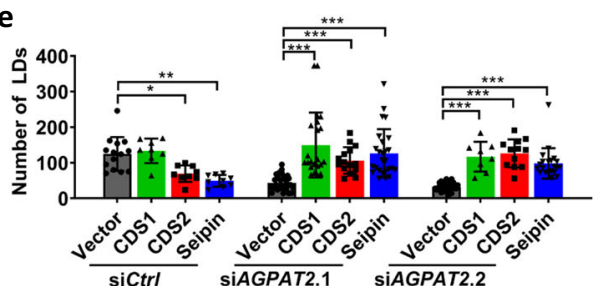

and CDS1/2 may form specific stable complexes to facilitate the delivery of PA to CDS1/2. To test this hypothesis, we carried out metabolic flux analyses using ${ }^{13} \mathrm{C}$-oleate as a tracer. We first conducted a time course experiment to assess ${ }^{13} \mathrm{C}$-oleate incorporation into phospholipids and TAGs (Supplementary Fig. 6a, b). We then conducted further analyses at the $8 \mathrm{~h}$ time point. Knocking down AGPAT2 reduced oleate incorporation into PI by
Fig. 3 Supersized LDs in AGPAT2-depletion cells can be rescued by reducing PA. a LD morphology after knocking down DGAT1 or DGAT2 in AGPAT2-deficient cells HeLa cells by siRNA. Cells were treated with $400 \mu \mathrm{M}$ oleic acid for $18 \mathrm{~h}$. Blue represents DAPI staining, and green represents BODIPY staining. Bars $=5 \mu \mathrm{m}$. $\mathbf{b}$ Quantification of LD diameters as shown in (a). mean $\pm \mathrm{SD}$; ${ }^{\star \star \star \star} p<0.0001 ;{ }^{*} p<0.05$, two-way ANOVA, $n=50$ cells examined over three biologically independent experiments. c LD morphology after overexpressing hCDS1-mCherry, hCDS2-mCherry and mCherry-hSeipin in AGPAT2 knockdown HeLa cells. Cells were treated with $400 \mu \mathrm{M}$ oleic acid for $18 \mathrm{~h}$. Green represents BODIPY staining and red represents mCherry expression. Bars $=10 \mu \mathrm{m}$. $\mathbf{d}$ and $\mathbf{e}$ Distribution of LDs according to diameters (d) and the number of LDs (e) after the overexpressing mCherry tagged-CDS1, CDS2 and seipin in control and AGPAT2-deficient cells. $\mathbf{d}$ LD size was represented by red $(>3 \mu \mathrm{m})$, green $(2-3 \mu \mathrm{m})$, white $(1-2 \mu \mathrm{m})$ and black $(0-1 \mu \mathrm{m})$. e mean $\pm S D ;{ }^{\star \star \star} p<0.001$; ${ }^{\star \star} p<0.01$; ${ }^{\star} p<0.05$, two-way ANOVA, $n=15-30$ cells examined over three biologically independent experiments.

1.7-fold and to a lesser extent, into PG, while incorporation into TAG increased by $\sim 40 \%$ (Fig. 6a). Conversely, overexpressing AGPAT2 increased oleate incorporation into PG (by 100\%) and PI (by $\sim 30 \%$ ) and reduced the flux to TAG by 3.3-fold (Fig. 6b). As a control, overexpressing AGPAT1 had a modest effect on the flux to PG and PI (Supplementary Fig. 6c). The rate of ${ }^{13} \mathrm{C}$-oleate incorporation into different lipids was shown in Supplementary Fig. 6d, e.

AGPAT2-deficient liver has reduced CDS2 protein level and CDS activity in vivo. To confirm our findings on the specific functional connection between AGPAT2 and CDS1/2 in vivo, we generated a liver-specific AGPAT2 knockout mouse (A2LKO mice) by CRISPR/Cas9-mediated gene editing. The strategy used to disrupt Agpat2 in mice is shown in Supplementary Fig. 7a: two Lox $P$ sites were introduced into the Agpat2 locus, sandwiching exons 2 and 4. Homozygous Agpat $2^{\mathrm{fl} / \mathrm{fl}}$ mice were then crossed with transgenic mice expressing Cre recombinase under the control of the albumin promoter. The resulting Agpat $2^{\mathrm{fl} /+} \mathrm{Alb}^{-\mathrm{creTg} / 0}$ progeny was then crossed with Agpat $2^{\mathrm{fl} / \mathrm{fl}}$ mice to generate the A2LKO mice. Littermates lacking the Cre gene $\left(\right.$ Agpat $\left.^{\mathrm{fl} / \mathrm{fl}}\right)$ were used as controls and referred to as WT. The successful genomic disruption of Agpat 2 was confirmed by the absence of the Agpat2 mRNA transcript and protein using western blotting and real-time quantitative PCR (Fig. 7a; Supplementary Fig. 7b). The level of PA in A2LKO liver increased almost three-fold (Fig. 7b), consistent with previous reports using other cell types/tissues ${ }^{11-13}$. Distinct from the global Agpat2-deficient $\left(\right.$ Agpat $\left.^{-/-}\right)$mice, the A2LKO mice have normal brown and white adipose tissue weight (BAT and WAT) (Supplementary Fig. 7c, d). Food intake, body weight, liver and gastrointestinal weight of the A2LKO mice also appear normal (Supplementary Fig. 7e-h). Expression analyses showed little change in other AGPATs, CDS1/2 and most key metabolic genes in the liver of A2LKO mice except a mild (50\%) increase in ACC2 (Supplementary Fig. 7i).

There was an increase of TAG but not total cholesterol (TC) in the liver of A2LKO mice fed either chow or high fat diet (HFD) (Fig. 7c, d). There also appeared to be slightly more and larger LDs in A2LKO liver on chow diet (Supplementary Fig. 7j, k). Notably, the liver of A2LKO mice on HFD appeared pale and enlarged, and the size of LDs increased dramatically, consistent with data from cell lines (Fig. 7e-h). Most importantly, the levels of hepatic CDS2 protein and hepatic CDS activity were significantly reduced in A2LKO mice (Fig. 7a and i). Moreover, oleate treatment of primary hepatocytes isolated from A2LKO mice resulted in enlarged LDs, which was reversed by 


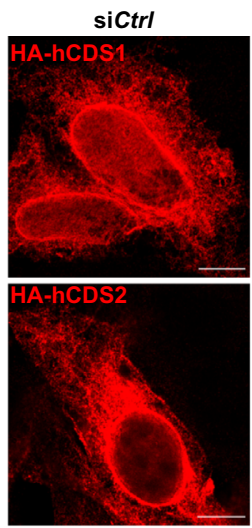

SiAGPAT2.1

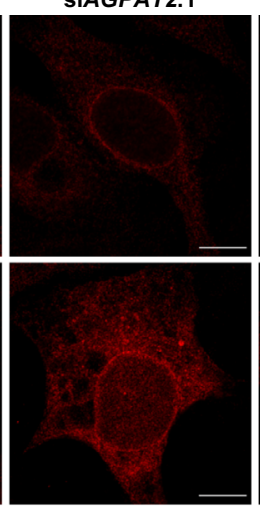

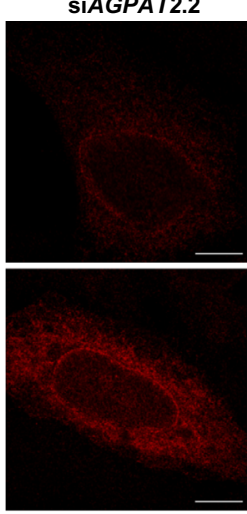

b

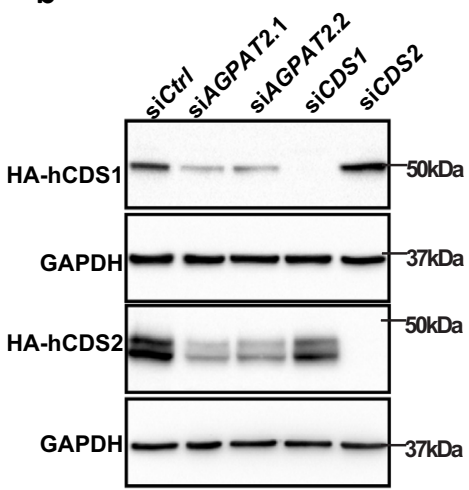

e

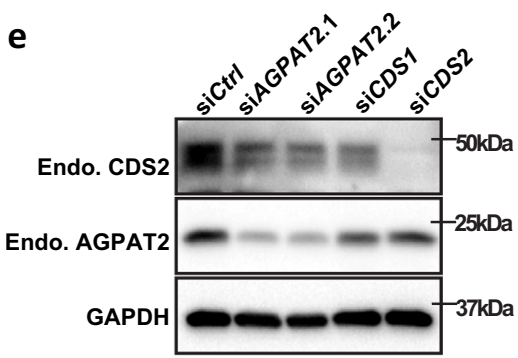

d

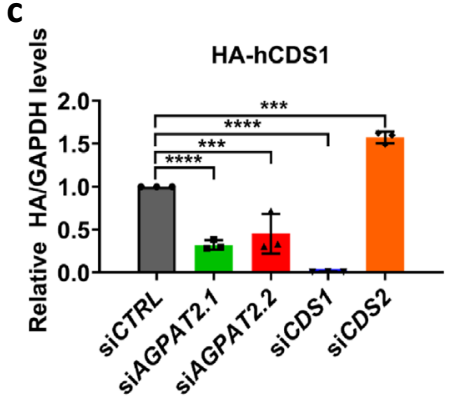

f

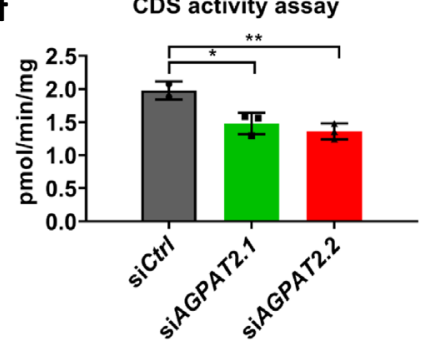

d

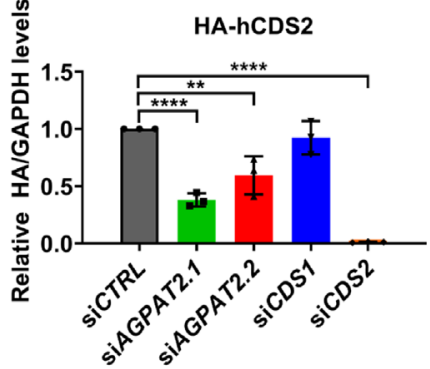

g

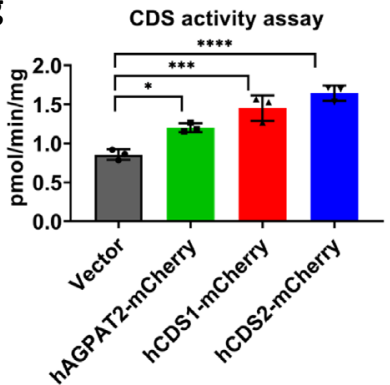

h

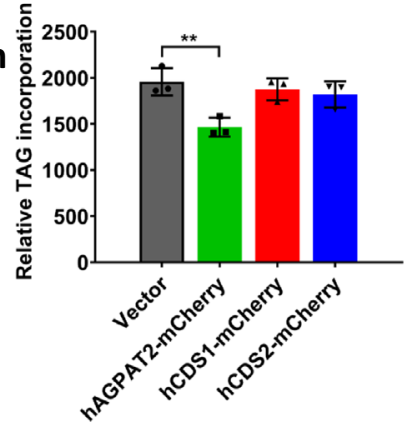

Fig. 4 Reduced CDS1/2 protein level and activity in AGPAT2-deficient cells. a The fluorescence intensity of HA-hCDS1/2 in control and AGPAT2 knockdown Huh7 cells. Bars: $10 \mu \mathrm{m}$. b Immunoblot of HA-tagged CDS1 and CDS2 in control, AGPAT2, CDS1 and CDS2 knockdown Huh7 cells. c, d Quantitation of (b) (mean \pm SD; one-way ANOVA ${ }^{\star \star \star \star} p<0.0001, n=3$ biologically independent experiments). e Immunoblot of endogenous CDS2 and AGPAT2 protein in control, AGPAT2 and CDS2 knockdown Huh7 cells. $\mathbf{f}$ CDS activity in control and AGPAT2 knockdown Huh7 cells. CDS activity assay was performed by using $\left[{ }^{3} \mathrm{H}\right]$ cytidine 5 '-phosphate, egg PA and $0.05 \mathrm{mg}$ membrane fractions from Huh7 transiently transfected with control or AGPAT2 siRNA. The radioactive products were measured by a scintillation counter (mean \pm SD; one-way ANOVA, ${ }^{\star \star} p<0.01,{ }^{*} p<0.05, n=3$ biologically independent experiments). $\mathbf{g}$ CDS activity in HeLa cells transiently transfected with mCherry-vector, hAGPAT2-mCherry, hCDS1-mCherry or hCDS2mCherry (mean $\pm \mathrm{SD}$; one-way ANOVA, ${ }^{\star \star \star} p<0.001,{ }^{\star \star} p<0.01,{ }^{\star} p<0.05, n=3$ biologically independent experiments). $\mathbf{h}$ TAG incorporation rate was measured by treating HeLa cells transiently transfected with mCherry-vector, hAGPAT2-mCherry, hCDS1-mCherry or hCDS2-mCherry with $1 \mu \mathrm{Ci}\left[{ }^{14} \mathrm{C}\right]$ oleate for $30 \mathrm{~min} .{ }^{14} \mathrm{C}$-labelled TAG was extracted, separated, and visualized by Typhoon FLA 9500 phosphor imager (mean \pm SD; one-way ANOVA, ${ }^{\star \star} p<0.01, n=5$ biologically independent experiments).

overexpressing mCherry-CDS2 (cells with red signal) (Fig. 7j, k). Overall, these results are consistent with data from cell lines and pull-down assays, and further support the functional relationship between AGPAT2 and CDS1/2.

\section{Discussion}

The mammalian genome encodes five putative AGPAT isoforms that catalyze a key step in the synthesis of phospholipids and TAGs: the acylation of LPA to PA. AGPAT2 is the only AGPAT isoform whose loss-of-function mutations are associated with $B S C L 1 / C G L 1^{9}$. It has been puzzling that the direct product of AGPAT2, PA, is increased in AGPAT2-deficient cells ${ }^{11-13}$. Our results from cellular studies, liver specific AGPAT2 knockout mice as well as biochemical analyses reveal that: 1. AGPAT2 regulates the biogenesis of cytoplasmic LDs; 2 . The stability and activity of CDS enzymes are decreased in AGPAT2-deficient cells and mouse liver, contributing to the increased PA under AGPAT2 deficiency; 3. AGPAT2 and CDS1/2 form a stable functional complex possibly to channel PA for the synthesis of CDP-DAG and phospholipids of the CDP-DAG branch, e.g., PI and PG. Most importantly, our results imply substrate channeling at a major branch point of the glycerol-3-phosphate pathway.

The immediate product of AGPAT2, PA, sits at a metabolic branch point for the synthesis of all phospholipids and TAGs 


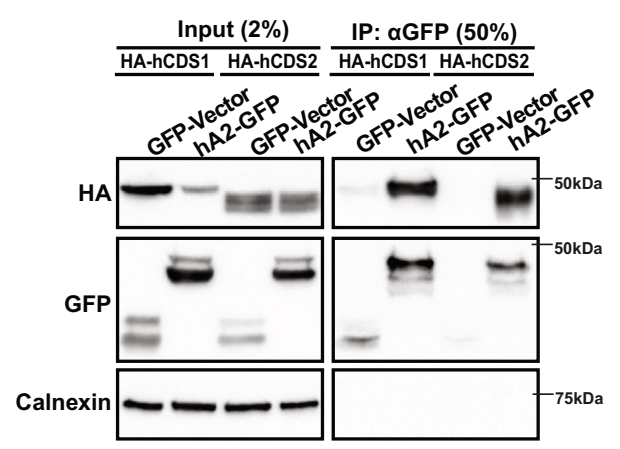

b
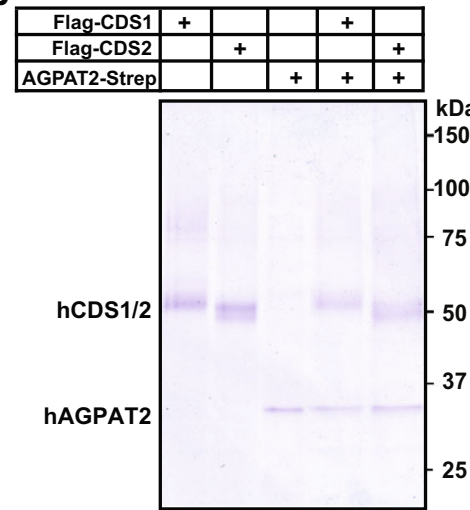

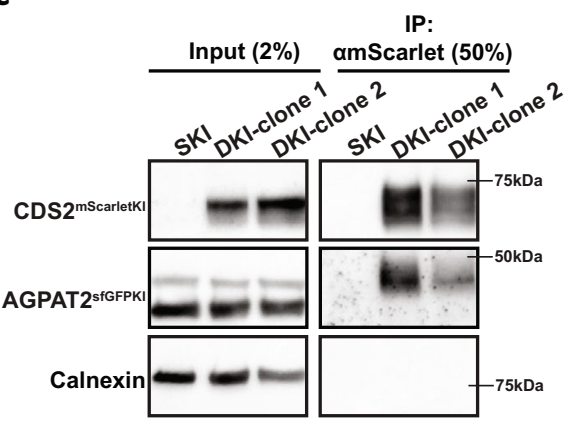

d
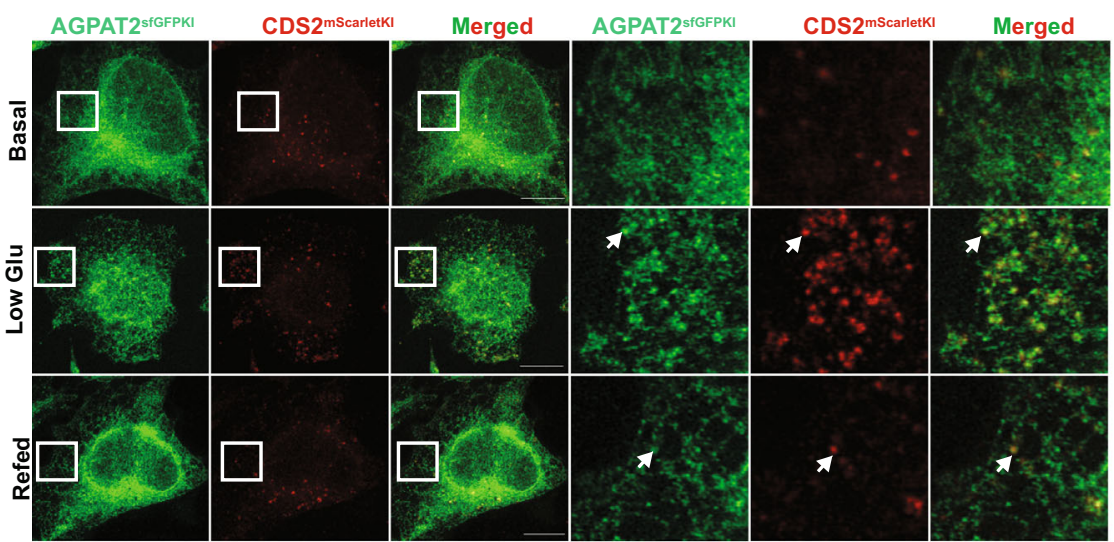

e

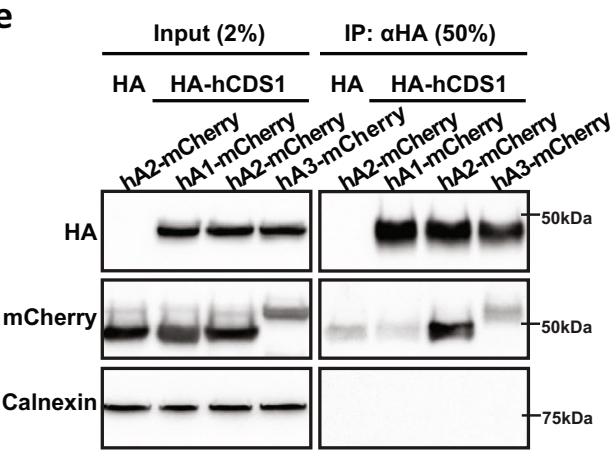

f

g
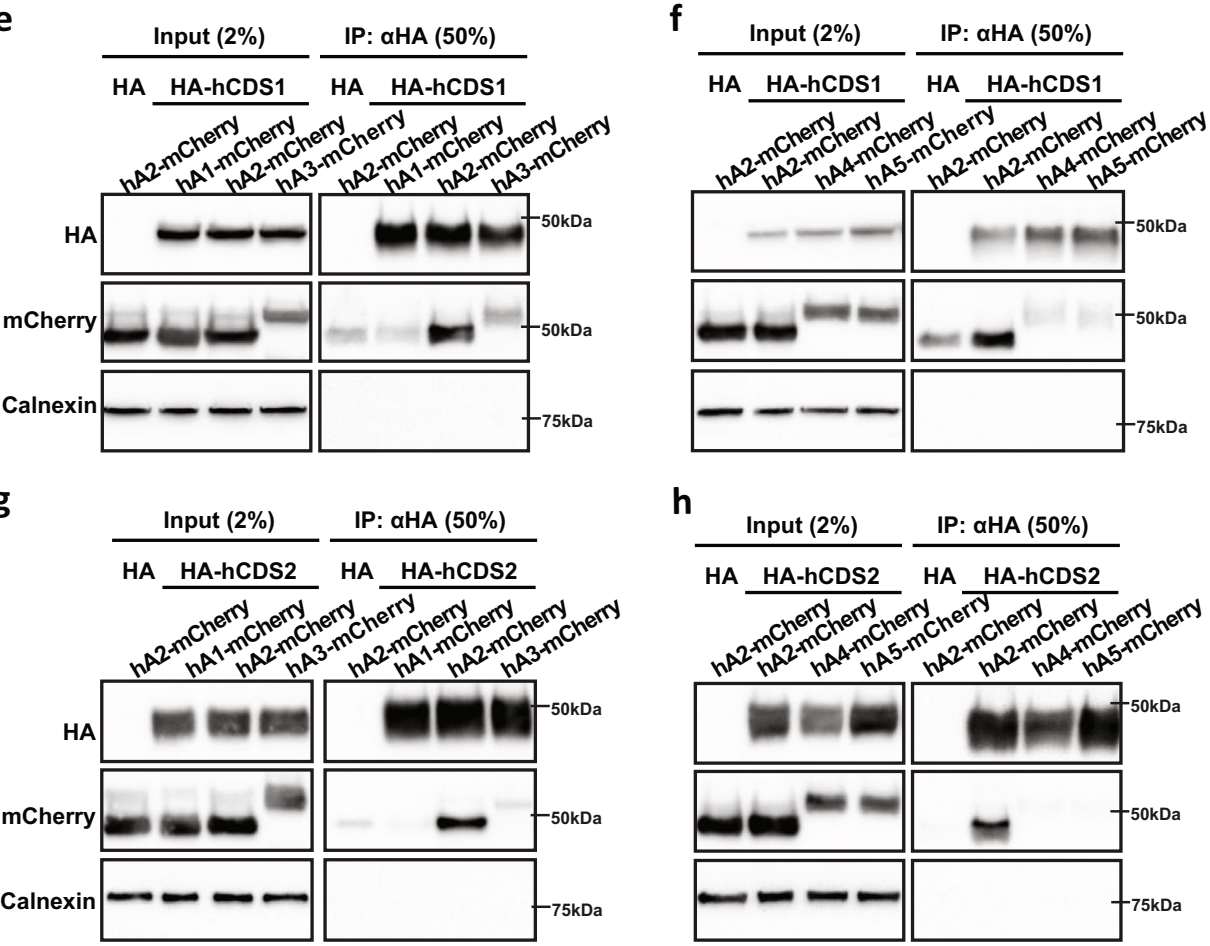

h

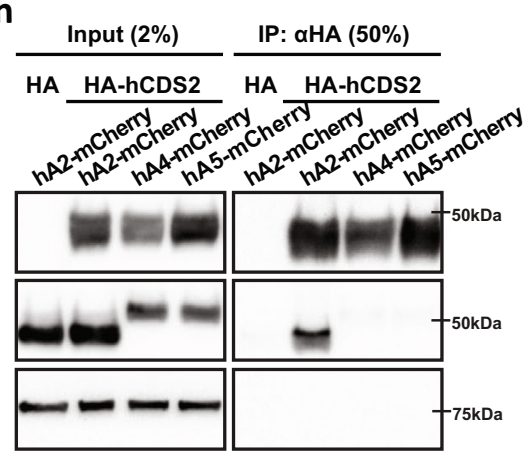

(Fig. 1a). PA can be de-phosphorylated into DAG by PAPs (i.e. the lipins) for the synthesis of PC, PE, PS and TAG ${ }^{35,36}$; PA can also be converted into CDP-DAG through the action of CDS1/2 for the synthesis of $\mathrm{PI}$, and possibly $\mathrm{PG}$ and $\mathrm{CL}^{8,32}$. Little is known about how the flux through this important metabolic branch point is regulated. Substrate channeling plays a key role in controlling flux at network branch points: the channeled metabolic intermediate is transferred directly from one enzyme to the next of the same biosynthetic pathway and is therefore prevented from being used by competing branch-point reactions ${ }^{33,34}$. The channeling is often facilitated by the formation of specific enzyme assemblies/protein complexes. An AGPAT2-CDS1/2 complex would channel PA towards the synthesis of CDP-DAG, thereby decreasing its reaction with PAPs 
Fig. 5 AGPAT2 and CDS1/2 physically interact. a Co-immunoprecipitation assay showing the interaction between AGPAT2-GFP and HA-CDS1/2 in HEK296E cells. $n=3$ biologically independent experiments. b Strep-tagged AGPAT2 and Flag-tagged CDS1 or CDS2 were expressed in HEK293F cells alone or together as indicated, followed by either a one-step affinity purification by anti-Flag or anti-Strep or a two-step affinity purification by anti-Flag and then anti-Strep. The Coomassie blue-stained gel shows purification of CDS1, CDS2 and AGPAT2, and co-purification of CDS1/2 and AGPAT2. $n=3$ biologically independent experiments. c Co-immunoprecipitation assay showing the interaction between endogenous AGPAT2-sfGFP and CDS2-mScarlet in HeLa cells. AGPAT2 or CDS2 was tagged at their genomic locus with sfGFP or mScarlet by CRISPR. SKI: single sfGFP knockin at AGPAT2. DKI: mScarlet knockin at CDS2 and sfGFP knockin at AGPAT2. $n=3$ biologically independent experiments. $\mathbf{d}$ Confocal imaging of fixed HeLa cells showing AGPAT2 and CDS2 tagged at their genomic loci with sfGFP and mScarlet, respectively. Cells were treated with high glucose DMEM (basal) or low glucose DMEM for $48 \mathrm{~h}$ or re-incubation with high glucose DMEM (Refed) for another $24 \mathrm{~h}$. Bars $=10 \mu \mathrm{m} . n=3$ biologically independent experiments. e Coimmunoprecipitation of mCherry-tagged AGPAT1, 2, 3 and HA-tagged CDS1 from transfected HEK293E lysates. $n=3$ biologically independent experiments. $\mathbf{f}$ Co-immunoprecipitation of mCherry-tagged AGPAT2, 4, 5 and HA-tagged CDS1 from transfected HEK293E lysates. $n=3$ biologically independent experiments. $\mathbf{g}$ Co-immunoprecipitation of mCherry-tagged AGPAT1, 2, 3 and HA-tagged CDS2 from transfected HEK293E lysates. $n=3$ biologically independent experiments. h Co-immunoprecipitation of mCherry-tagged AGPAT2, 4, 5 and HA-tagged CDS2 from transfected HEK293E lysates. $n=3$ biologically independent experiments.

for DAG synthesis. Indeed, AGPAT2 is the only AGPAT isoform that specifically and directly interacts with CDS1 and CDS2. Strikingly, CDS proteins became unstable and CDS activity was significantly reduced under AGPAT2 deficiency, in both cell lines and mouse liver. Moreover, overexpressing AGPAT2 increased CDS activity, and reduced oleate incorporation into TAG. Consistently, metabolic flux analyses showed that the rate of oleate incorporation into PG/PI (the CDS branch) was increased upon overexpressing and decreased upon knocking down AGPAT2. It is also worth noting that the steady-state concentrations of PI and PG were significantly reduced in AGPAT2-deficient mouse embryonic fibroblasts ${ }^{12}$, consistent with our present observations. Together, our data provide physical and functional evidence that AGPAT2 and CDS1/2 form complexes to promote the flux of PA into the CDP-DAG pathway. Without AGPAT2, CDS activity is reduced, causing accumulation of $\mathrm{PA}$, aberrant $\mathrm{LD}$ formation and impaired adipogenesis ${ }^{30,32}$. Although CDS1/2 and AGPAT2 can form rather stable complexes which can be co-purified in large quantity, we were unable to resolve the structures of the two complexes at this time despite multiple attempts. While CDS1 and CDS2 can both interact with AGPAT2, we were unable to detect a stable interaction between CDS1 and CDS2 (data not shown). Thus, AGPAT2 may associate with CDS1 or CDS2 separately, and the resulting distinct complexes may operate at different regions of the ER, and/or catalyze the synthesis of different CDP-DAG species, and/or function under different conditions. Future efforts are required to obtain insights into the structural details of the AGPAT2-CDS1/2 complexes and their specific functions.

The finding that AGPAT2's product, PA, is increased in AGPAT2-deficient cells and tissues has been confusing and paradoxical. We show here that the reduced CDS activity under AGPAT2 deficiency may be at least partially responsible for the increase in PA. Indeed, overexpressing CDS1/2 reduced the accumulation of $\mathrm{PA}$ and restore normal LD formation in AGPAT2-deficient cells. Although CDS1/2 can clearly form functional complexes with AGPAT2, our results also suggest that $\mathrm{CDS} 1 / 2$ can use PA generated from other sources (e.g. other AGPATs, DAG kinases or phospholipase D) ${ }^{37}$. Under AGPAT2 deficiency, CDS activity and the flux of oleate to PG and PI were significantly but only moderately reduced, suggesting that PA from other sources is available for use by the remaining CDS enzymes. There also appears to be increased flux of oleate to PA synthesis in AGPAT2-deficient cells. Therefore, AGPAT2 deficiency may trigger a range of biochemical changes in addition to impaired CDS stability and activity, and the molecular basis for those changes requires further investigation.
Our results also reveal cell autonomous roles of AGPAT2 in LD formation. AGPAT2 deficiency delayed the maturation of initial LDs and formed supersized LDs after prolonged oleate treatment, phenotypes reminiscent of seipin deficiency 14,17,24,29,38. The delay in early LD lipidation in AGPAT2-deficient cell is not as severe as that in seipin knockout cells. It should be noted that while seipin was knocked out, AGPAT2 was knocked down, because the AGPAT2 knock-out cancer cell lines were very sick in our hands and therefore not used. Nevertheless, these results further connect BSCL1 (AGPAT2) with BSCL2 (seipin) beyond adipogenesis. As PA has been implicated in seipin function ${ }^{15-18,39}$, the increased PA in the ER may also underpin the effect of AGPAT2 deficiency on LD dynamics. Indeed, aberrant LD formation under AGPAT2 deficiency can be rescued by overexpressing CDS1/2 (consuming PA) or seipin (reducing PA production and increasing PA sequestration) ${ }^{16,17}$. Thus, our results highlight the role of non-bilayer lipids (e.g. PA) in LD biogenesis possibly by modulating the surface tension and curvature at sites of LD formation ${ }^{22}$. To investigate the function of AGPAT2 in vivo, we generated the liver specific A2LKO. The LD phenotypes in AGPAT2-deficient liver and primary hepatocytes are consistent with those observed in cell lines. Importantly, CDS activity was significantly reduced in A2LKO liver, and the LD phenotype in AGPAT2-deficient primary hepatocytes was rescued by overexpressing CDS2. Overall, these results suggest that the reduced CDS activity in AGPAT2-deficient hepatocytes led to accumulation of PA in the ER, forming enlarged cytoplasmic LDs.

Although our data from biochemical assays, cell line and mouse studies strongly support a role for AGPAT2 to promote the synthesis of CDP-DAG branch of phospholipids, there are some limitations. We could not directly visualize the channeling of PA towards CDP-DAG synthesis in the AGPAT2-CDS1/2 complex. This may be achievable after the structures of AGPAT2-CDS1/2 complexes are resolved. There is also some limitation in our flux studies because ${ }^{13} \mathrm{C}$-oleate can be incorporated into pre-existing phospholipids by the deacylation-reacylation pathway. However, it should be noted that this pathway may be a minor contributor to ${ }^{13} \mathrm{C}$-oleate incorporation into phospholipids compared with the de novo synthesis pathway.

In summary, we provide strong evidence that AGPAT2 and CDS1/2 can form stable complexes which promote CDP-DAG synthesis. We also demonstrate a role for AGPAT2 in normal LD formation. Together, these results provide key insights into the regulation of the glycerol-3-phosphate pathway, unveil the molecular basis for the increase in PA under AGPAT2 deficiency and for the pathogenesis of $B S C L 1$, and open future avenues of investigation on how the metabolism of PA is controlled at a major metabolic branch point. 
a

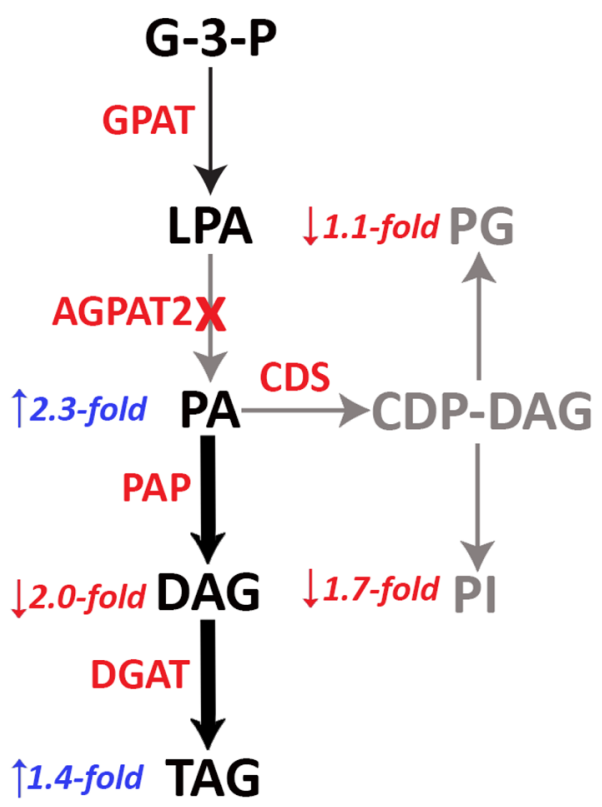

b

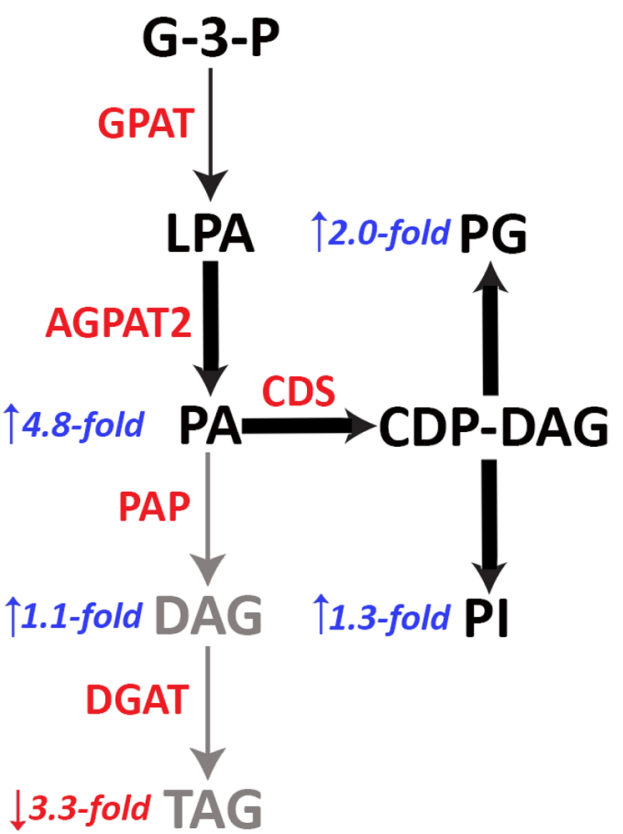

Fig. 6 AGPAT2 promotes oleate incorporation into phospholipids of the CDP-DAG branch. HeLa cells were transfected with AGPAT2 siRNA (a) or human AGPAT2-mCherry (b) for $24 \mathrm{~h}$. Cells were then treated with dialysed FBS for $16 \mathrm{~h}$ and then for $8 \mathrm{~h}$ with $10 \mu \mathrm{M}\left[\mathrm{U}^{13} \mathrm{C}\right]$-oleate (C18:1). ${ }^{13} \mathrm{C}$-labelled samples were analysed by LC-MS. Fold changes of oleate incorporation were indicated as blue (increase) and red (decrease) arrows.

\section{Methods}

Antibodies, chemicals, plasmids, and primers. Antibodies, chemicals and plasmids with source and catalogue numbers are described in Supplementary Table 1. Primers used for cloning, for PCR and for knock-in (KI) are listed in Supplementary Tables $2-4$

Cell culture, RNAi, and transfection. AML12, HeLa and Huh7 cells were grown in Dulbecco's modified Eagle medium (DMEM) with 10\% foetal bovine serum
(FBS) and $1 \%$ penicillin-streptomycin-glutamine (PSG). Cells were maintained in $37{ }^{\circ} \mathrm{C}$ incubator with $5 \% \mathrm{CO}_{2}$. Medium was changed every 2 days. AML12 cells were grown in DMEM:nutrient mixture F-12 (DMEM/F12) with 10\% FBS, $1 \%$ insulin-transferrin-selenium (ITS-G) and $40 \mathrm{ng} / \mathrm{mL}$ dexamethasone. 3T3-L1 cells were grown in DMEM with $10 \%$ Newborn calf serum (NCS) and 1\% PSG.

Transient plasmid transfection was carried out by using Lipofectamine LTX. Plasmid DNA and Lipofectamine LTX were diluted in Opti-MEM ${ }^{\mathrm{m}}$ I Reduced Serum Media separately, followed by $5 \mathrm{~min}$ incubation in room temperature. The mixture was incubated for another $10 \mathrm{~min}$ after mixed and added to the cells culture. Cells were harvested $48 \mathrm{~h}$ post transfection.

Transient small interfering RNA (siRNA) transfection was carried out by using Lipofectamine RNAiMAX. $20 \mu \mathrm{M}$ siRNA and RNAiMAX (twice the volume of siRNA) were diluted in Opti-MEM ${ }^{\mathrm{mw}}$ I Reduced Serum Media separately, followed by $5 \mathrm{~min}$ incubation at room temperature. The mixture was incubated for another $20 \mathrm{~min}$ and added to cell culture. Cells were harvested $48 \mathrm{~h}$ post transfection.

\section{Generation of KI cells with CRISPR/Cas12a-mediated genome editing.} C-terminally mCherryTag-tagged PLIN3 was generated by CRISPR/Cas9 gene editing ${ }^{40}$. HeLa cells were simultaneously transfected by Lipofectamine ${ }^{\text {ix }}$ LTX Reagent with PLUS ${ }^{\text {w }}$ Reagent (\#15338100, Thermo Fisher Scientific) with Megamer ${ }^{\oplus}$ Single-Stranded DNA Fragments (Integrated DNA technologies) containing arms with 100-nucleotide-long homology upstream and downstream of the target site and gRNA targeting upstream of stop codon. The single-stranded DNA fragments and gRNA information are described in Supplementary Table 3. C-terminally superfolderGFP-tagged AGPAT2 and mScarlet-tagged CDS2 was generated by CRISPR/Cas12a gene editing method ${ }^{41}$. A PCR cassette, containing sfGFP/mScarlet, a Cas12a CRISPR RNA and $\sim 100$ bp homology arms, were amplified from pMaCTag-P06 by Phusion ${ }^{\text {tut }}$ high-fidelity DNA polymerase (\#F530L, Thermo Fisher Scientific). Primers were designed by http://www.pcrtagging.com/. HeLa cells were simultaneously transfected by Lipofectamine ${ }^{\text {it }}$ LTX Reagent with PLUS ${ }^{\text {me }}$ Reagent (\#15338100, Thermo Fisher Scientific) with equal amount of pcDNA3.1-hLbCpf1(TYCV) and PCR cassettes.

For PLIN3-mCherry KI, CDS2-mScarlet KI and AGPAT2-sfGFP KI, cells were then selected with $2 \mu \mathrm{g} / \mathrm{mL}$ puromycin for $48 \mathrm{~h}$ and recovered for 1-2 weeks in the absence of puromycin. Single-cell FACS sorting was performed by BD FACSMelody ${ }^{\text {ma }}$ Cell Sorter (BD Biosciences) at the flow cytometry (UNSW). 561 and $488 \mathrm{~nm}$ laser were used to sort mCherry and sfGFP-positive cells, respectively. To validate the insertion of tags, target regions were amplified by PCR and sequenced.

Viral stable transduction. LentiX-293T cells were used for lentiviral stable knockdown. LentiX-293T cells were plated at $2 \times 10^{6}$ cells per $10 \mathrm{~cm}$ dish $24 \mathrm{~h}$ prior to transfection. Cells were transfected by using Lipofectamine LTX. For lentiviral production, $10 \mu \mathrm{g}$ AGPAT2 shRNA, $2.970 \mu \mathrm{g}$ pMD.G, $5.294 \mu \mathrm{g}$ pMDLg/pRRE and $1.848 \mu \mathrm{g}$ pRSVrev were transfected to LentiX-293T cells, followed by incubation in $37^{\circ} \mathrm{C}$ with $5 \% \mathrm{CO}_{2}$ for $48 \mathrm{~h}$. Media with virus were collected from LentiX-293T cells and filtered through a $0.45 \mu \mathrm{M}$ filter, followed by the addition of $8 \mu \mathrm{g} / \mathrm{mL}$ polybrene. Lentivirus titre was tested by Lenti-X GoStix Plus. The filtered viral media was then added to the 3T3-L1 adipocytes and incubated for $24 \mathrm{~h}$.

Adipocyte differentiation. To induce adipocyte differentiation, 3T3-L1 preadipocytes were grown in DMEM with 10\% NCS and 1\% PSG until 10\% confluency. Two days post-confluency, differentiation was stimulated by using DMEM containing $10 \%$ FBS, $1 \%$ PSB and supplemented with insulin $(10 \mu \mathrm{g} / \mathrm{mL})$, dexamethasone $(1 \mu \mathrm{M})$ and isobutylmethyxantine (IBMX) $(0.5 \mathrm{mM})$. An additional 2 days later, cells were grown in DMEM/FBS/PSG with insulin $(10 \mu \mathrm{g} / \mathrm{mL})$. Media (DMEM/FBS/PSG) was refreshed every 2 days until the end of the differentiation.

Cell proliferation assay. 3T3-L1 cells were grown and differentiated in a 96-well plate. Cells were transduced with control and AGPAT2 shRNA lentivirus at day 6 of differentiation. Cell proliferation Assay was performed at day 8 of differentiation by using CellTiter $96^{\circ}$ AQueous One Solution (Promega) according to manufacturer's protocol.

LDs studies. For fixed samples, cells were treated with $400 \mu \mathrm{M}$ oleate-coupled BSA in DMEM/FBS/PSG for $18 \mathrm{~h}$. Cells were rinsed twice with PBS and postfixed with 4\% paraformaldehyde for $15 \mathrm{~min}$ at room temperature. Cells were rinsed three times with PBS before and after staining with freshly prepared $1 \mu \mathrm{g} / \mathrm{mL}$ BODIPY 493/503 (Thermofisher scientific) for $15 \mathrm{~min}$. For immunofluorescence staining, cells were subsequently permeabilized with $0.2 \%$ TritonX-100 in PBS for $15 \mathrm{~min}$ at $\mathrm{RT}$ after fixation, and then blocked by incubation with 3\% (w/v) BSA in PBS for $1 \mathrm{~h}$ at RT. Cells were than incubated with primary antibody and secondary antibody in $3 \%(\mathrm{w} / \mathrm{v})$ BSA for $1 \mathrm{~h}$ at RT, with three $5 \mathrm{~min}$ PBS washes in between. Coverslips were then mounted onto slides by using ProLong ${ }^{m}$ Gold Antifade Mountant with DAPI (Thermofisher Scientific). All images were obtained by ZEISS LSM 900 with Airyscan microscopy (Carl Zeiss, Jena, Germany) with $\times 63$ Plan Apochromat (1.4 NA) oil objective. Acquired images were quantified by ImageJ software. 
a

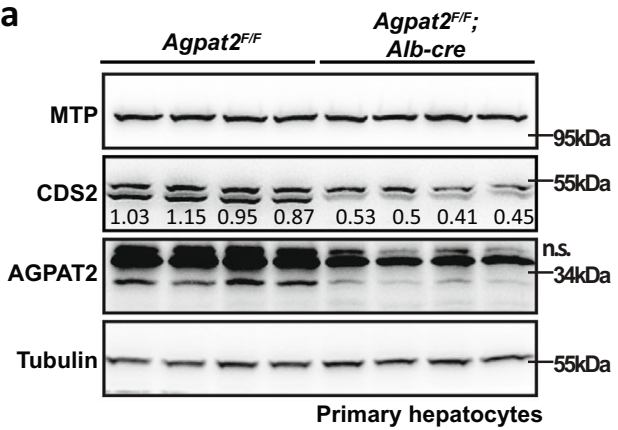

d

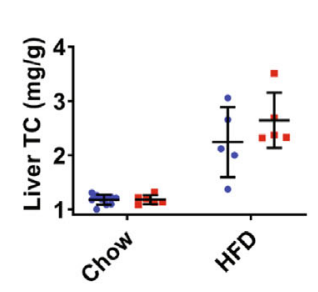

e b
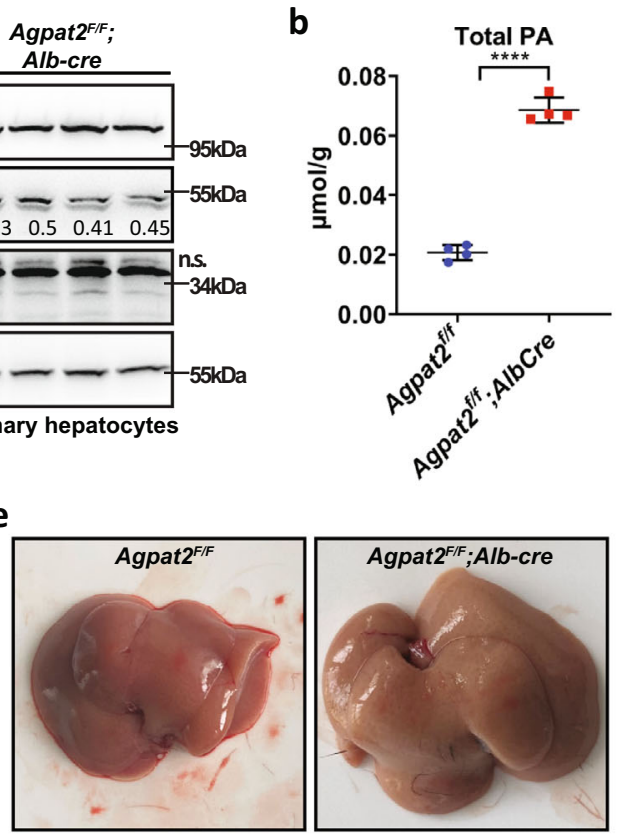

C

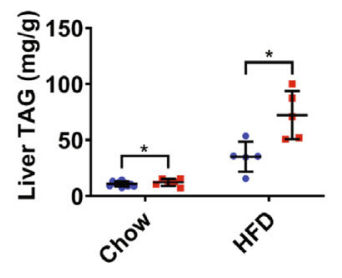

f

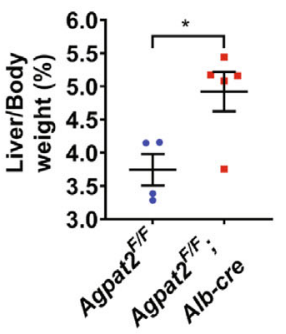

g

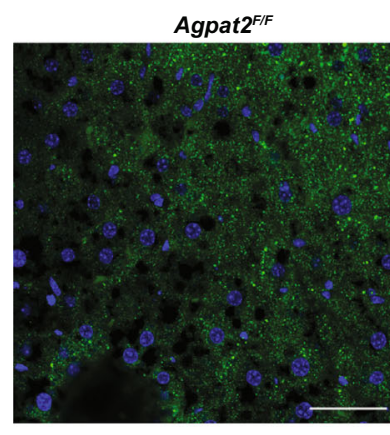

Agpat2 $^{\mathrm{F} / F} ;$ Alb-cre

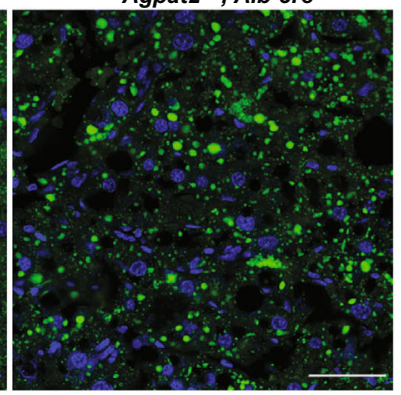

h

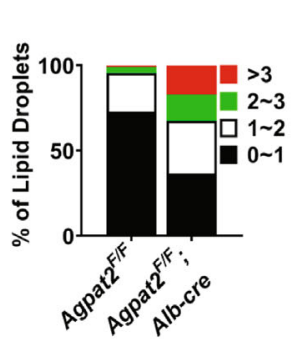

i

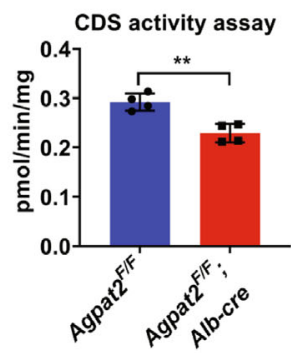

j

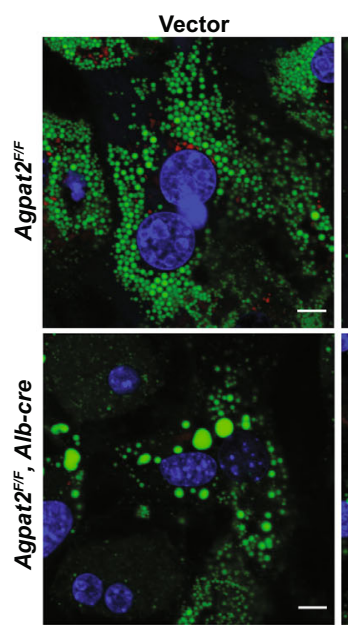

mCherry-mCDS2
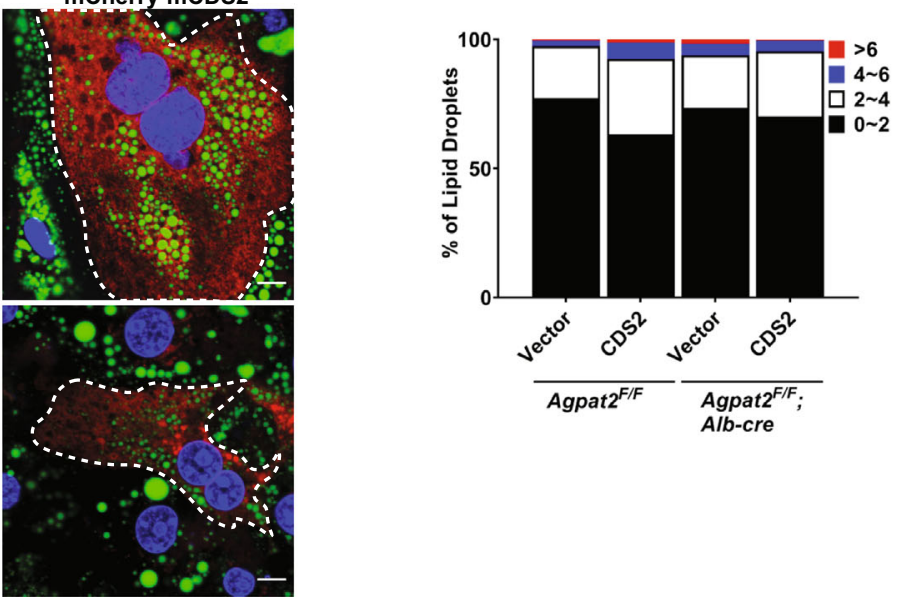

Fig. 7 Ablation of AGPAT2 from mouse liver reduces hepatic CDS activity. a Western blotting of MTP, CDS2 and AGPAT2 from primary hepatocytes isolated from WT (Agpat2f/f $n=4)$ and liver-specific AGPAT2 knockout mice (A2LKO/Agpat2f/f, Alb-cre, $n=4)$. b Total PA levels of WT ( $n=4)$ and A2LKO $(n=4)$ mice fed chow diet. $\mathbf{c}$ Liver TAG and $\mathbf{d}$ total cholesterol (TC) of WT $(n=6-9)$ and A2LKO $(n=5-7)$ mice fed chow or HFD. e Liver morphology and $\mathbf{f}$ the ratio of liver to body weight from WT and A2LKO mice fed HFD. $\mathbf{g}$ BODIPY-stained liver sections from WT and A2LKO mice fed HFD. Bars: $50 \mu \mathrm{m}$. h Distribution of LDs of different diameters as shown in $(\mathbf{g})$. i CDS activity of WT $(n=4)$ and A2LKO liver $(n=4)$. j Primary hepatocytes from WT and A2LKO chow-fed mice were transfected with mCherry-CDS2 and stained with BODIPY. Dashed line indicates cells transfected with mCherry-CDS2. $\mathbf{k}$ Distribution of LDs according to diameters as shown in (j). $\mathbf{b}-\mathbf{d}, \mathbf{f}$ and $\mathbf{i}$ mean \pm SD; two-tailed unpaired $t$ test. ${ }^{\star} p<0.05,{ }^{\star \star} p<0.01$, $\star \star \star \star x p<0.0001$. 
Live cell imaging. For live-cell imaging, HeLa cells were cultured in in DMEM/ $10 \%$ FBS/PSG. Pre-LDs were removed by starving the cells with $1 \%$ LPDS in DMEM for $16 \mathrm{~h}$. All live cell imaging experiments were performed at $+37^{\circ} \mathrm{C}, 5 \%$ $\mathrm{CO}_{2}$ in FluroBrite DMEM supplemented with $1 \%$ LPDS and ProLong Live Antifade (Thermofisher Scientific). Equal volumes of media containing two times concentrated oleate-coupled BSA $(800 \mu \mathrm{M})$ and BODIPY (1:5000) was added to the dish immediately before image acquisition. HILO microcopy was performed using Zeiss Elyra inverted microscope equipped with a high-sensitivity Andor iXon 897 EMCCD cameras fitted with a $\times 63$, NA 1.4 Plan-Apochromat lens. Images were obtained every $1.26 \mathrm{~s}$. Acquired live cells images were deconvoluted and quantified by custom MATLAB scripts.

SDS-PAGE and immuno-blotting. Cells were washed twice with ice-cold PBS and lysed by adding $0.1 \%$ SDS lysis buffer ( $1 \%$ Triton X-100, $0.1 \%$ SDS, $10 \mathrm{mM}$ Tris $\mathrm{pH}$ $7.4,100 \mathrm{mM} \mathrm{NaCl}, 1 \mathrm{mM}$ EDTA, $10 \%$ glycerol and complete protease Inhibitor Cocktail tablet, EDTA-Free). Cell lysates were then incubated on ice for $15 \mathrm{~min}$, followed by centrifuging at $18,000 \times g$ for $10 \mathrm{~min}$ at $4{ }^{\circ} \mathrm{C}$. Supernatant was then transferred to a new tube. Protein concentration was determined by using BCA protein assay kit. $40 \mu \mathrm{g}$ protein lysate was mixed with $2 \times$ Laemmli sample buffer and then loaded to $10 \%$ SDS-PAGE gel. Electrophoresis was run at $150 \mathrm{~V}$ for $1 \mathrm{~h}$. Proteins on the gel were then transferred to nitrocellulose membrane at $100 \mathrm{~V}$ for $1 \mathrm{~h}$. Membranes were blocked with 5\% (w/v) skim milk in TBST in room temperature for $1 \mathrm{~h}$, followed by incubating with primary antibody with appropriate dilution at $4{ }^{\circ} \mathrm{C}$ for $16 \mathrm{~h}$. After primary antibody incubation, membranes were wash with TBST three times for 5 min each and then incubated with secondary antibody at 1:5000 dilution in TBST for $1 \mathrm{~h}$ at room temperature. Membranes were washed again with TBST three times for $5 \mathrm{~min}$ each and developed by using enhanced chemiluminescence and BioRadChemiDoc XRS+ imager.

Co-immunoprecipitation. Protein lysates $(1 \mathrm{mg})$ were mixed with $25 \mu \mathrm{L} \mathrm{HA}$ agarose beads to a final volume of $500 \mu \mathrm{L}$ per sample by using co-IP lysis buffer (25 mM HEPES pH 7.4, $150 \mathrm{mM} \mathrm{NaCl}, 1 \mathrm{mM}$ EDTA, $10 \%$ glycerol, $1 \%$ DDM) to normalize. Protein-beads mixture was then gently rotated at $4{ }^{\circ} \mathrm{C}$ for $16 \mathrm{~h}$. Proteinbeads mixture was washed three times with $150 \mathrm{mM}$ wash buffer $(25 \mathrm{mM}$ HEPES $\mathrm{pH} 7.4,150 \mathrm{mM} \mathrm{NaCl}, 0.1 \% \mathrm{NP}-40$ ). Proteins were eluted by adding $30 \mu \mathrm{l}$ $2 \times$ Protein loading buffer without $\beta$-mercaptoethanol, followed by vortexing, and centrifuging at $18,000 \times g$ for $5 \mathrm{~min}$. Supernatant was then transferred to a new tube and $30 \mu \mathrm{l} 2 \times$ protein loading buffer (with $\beta$-mercaptoethanol) was added. Protein lysates were then subjected to SDS-PAGE and Western blot.

RNA extraction and quantitative real-time PCR. Total RNA was extracted using TRIzol $^{\mathrm{TM}}$ reagent. Mammalian cells were grown in $6 \mathrm{~cm}$ Petri dishes. Cells were washed with ice cold PBS once, followed by adding $1 \mathrm{~mL}$ TRIzol ${ }^{\mathrm{TM}}$ reagent and rocking for $5 \mathrm{~min} .200 \mu \mathrm{L}$ chloroform was added and the mixture was shaken vigorously. After incubating for $3 \mathrm{~min}$ in room temperature, the mixture was then centrifuged at $12,000 \times g$ for $15 \mathrm{~min}$ at $4^{\circ} \mathrm{C}$. After centrifugation, the top aqueous layer was transferred to a new tube and $0.5 \mathrm{~mL} 100 \%$ isopropanol was added. After 10 min incubation in room temperature, the mixture was then centrifuged again at $12,000 \times g$ for $10 \mathrm{~min}$ at $4{ }^{\circ} \mathrm{C}$. After centrifugation, supernatant was removed and $1 \mathrm{~mL} 75 \%$ ethanol was added, followed by vortexing for $15 \mathrm{~s}$. The sample was then subjected to centrifugation at $7500 \times g$ for $5 \mathrm{~min}$ at $4{ }^{\circ} \mathrm{C}$. This step was repeated twice. The RNA pellet was dried at $55^{\circ} \mathrm{C}$ for $10 \mathrm{~min}$ and dissolved in RNAse free water. cDNA synthesis was then performed by using $1 \mu \mathrm{g}$ RNA and high-capacity cDNA reverse transcription kit. cDNA was synthesized by one cycle of denaturation at $25^{\circ} \mathrm{C}$ for $10 \mathrm{~min}$, annealing and extension at $37^{\circ} \mathrm{C}$ for $120 \mathrm{~min}$ and enzyme deactivation at $75^{\circ} \mathrm{C}$ for $5 \mathrm{~min}$.

All qRT-PCR primers used in this project are listed in Supplementary Table 3. qRT-PCR was performed by using the KAPA SYBR FAST qRT-PCR kit. qRT-PCR samples were held at $95^{\circ} \mathrm{C}$ for $4 \mathrm{~min}$ then followed by 40 cycles of denaturation at $95^{\circ} \mathrm{C}$ for $10 \mathrm{~s}$, annealing at $60^{\circ} \mathrm{C}$ for $20 \mathrm{~s}$ and extension at $72^{\circ} \mathrm{C}$ for $20 \mathrm{~s}$. The mRNA expression levels were obtained by normalizing against house-keeping gene and comparing to control.

CDS1/2-AGPAT2 co-purification assay. The full-length cDNA of human CDS1, CDS2 and AGPAT2 were subcloned into the pCAG vector, tagged with an $\mathrm{N}$-terminal Flag tag for CDS1/2 and a C-terminal Strep tag for AGPAT2. The recombinant Flag-CDS1/CDS2 and AGPAT2-Strep were co-expressed in HEK293F cells. The cells were transiently transfected at a density of $2.0 \times 10^{6}$ cells per mL, using polyethyleneimine (PEI) (Polysciences). A 100-mL cell culture was transfected with $0.075 \mathrm{mg}$ of CDS1 or CDS2 plasmid, and $0.075 \mathrm{mg}$ of AGPAT2 plasmid. After $12 \mathrm{~h}$, the cell culture was supplemented with $10 \mathrm{mM}$ sodium butyrate, to boost protein expression. After another $48 \mathrm{~h}$, the cells were collected by centrifugation and were resuspended in buffer containing $25 \mathrm{mM}$ Tris $\mathrm{pH} 8.0,150 \mathrm{mM}$ $\mathrm{NaCl}$, and protease inhibitor cocktails (Amresco). Membranes were solubilized at $4{ }^{\circ} \mathrm{C}$ for $2 \mathrm{~h}$ with $1 \%(\mathrm{w} / \mathrm{v}) \mathrm{GDN}$ (Anatrace). After centrifugation at $20,000 \times g$ for $45 \mathrm{~min}$, the supernatant was applied to anti-Flag G1 affinity resin (GenScript). The resin was rinsed with buffer W $(25 \mathrm{mM}$ Tris $\mathrm{pH} 8.0,150 \mathrm{mM} \mathrm{NaCl}, 0.02 \%(\mathrm{w} / \mathrm{v})$ GDN, $5 \mathrm{mM}$ ATP, $5 \mathrm{mM} \mathrm{MgCl}_{2}$ ), and bound protein was eluted with buffer E1 (25 mM Tris pH 8.0, $150 \mathrm{mM} \mathrm{NaCl}, 0.02 \%$ (w/v) GDN, $200 \mu \mathrm{g} / \mathrm{mL}$ Flag peptide).
The eluent was then applied to Strep-Tactin resin (IBA Lifesciences). After rinsing with buffer W, bound protein was eluted with buffer E2 $(25 \mathrm{mM}$ Tris $\mathrm{pH} 8.0$, $150 \mathrm{mM} \mathrm{NaCl}, 0.02 \%(\mathrm{w} / \mathrm{v}) \mathrm{GDN}, 50 \mathrm{mM}$ Biotin). The eluent was analysed by SDS-PAGE and stained with Coomassie blue dyes.

CDS activity assay. CDS activity assay was performed as described ${ }^{42}$. Huh7 cells were resuspended in ice cold lysis buffer $(50 \mathrm{mM}$ Tris- $\mathrm{HCl}(\mathrm{pH} 8.0), 50 \mathrm{mM} \mathrm{KCl}$, $0.2 \mathrm{mM}$ ethylene glycol tetraacetic acid (EGTA), and 1/100 (v/v) protease inhibitor cocktail) and lysed by 30 passes through homogenizer. Nuclei and unbroken cells were removed by centrifugation at $1000 \times g$ for $10 \mathrm{~min}$ at $4^{\circ} \mathrm{C}$. Membrane fraction was then extracted by removing the supernatant and centrifuging at $100,000 \times g$ for $1 \mathrm{~h}$ at $4{ }^{\circ} \mathrm{C}$.

$200 \mu \mathrm{M}$ Egg PA was dried down by speediVac and resuspended in $125 \mathrm{mM}$ Tris- $\mathrm{HCl}$ (pH 8), $250 \mathrm{mM} \mathrm{KCl}, 12.75 \mathrm{mM}$ Triton X-100, $5 \mathrm{mg} / \mathrm{mL}$ BSA and $0.625 \mathrm{mM}$ DTT. CDS activity assay was performed in a $100 \mu \mathrm{l}$ reaction. $2 \mu \mathrm{l} \mathrm{MgCl}_{2}$ (1 M stock) was added to $40 \mu \mathrm{l}$ egg PA suspension, followed by $48 \mu \mathrm{l}$ of protein sample $(50 \mu \mathrm{g})$. The reaction was then initiated by adding $7.5 \mu \mathrm{l}$ of CTP $(200 \mu \mathrm{M}$ stock) pre-mixed with $2.5 \mu \mathrm{Ci} \mathrm{CTP}\left[5^{\prime}-3 \mathrm{H}\right]$ and incubated for $10 \mathrm{~min}$ at $30^{\circ} \mathrm{C}$. This was then terminated by adding $375 \mu \mathrm{l}$ chloroform:methanol (1:2), followed by adding $125 \mu \mathrm{l}$ chloroform and $125 \mu \mathrm{l}$ water. The mixture was vortexed and centrifuged in $1000 \times g$ for $5 \mathrm{~min}$. The lower organic phase was recovered and washed with the synthetic top phase, $125 \mu \mathrm{l}$ methanol and water (1:0.9). The lower organic phase was evaporated in a fume hood prior to counting the radioactivity in a scintillation counter.

${ }^{14} \mathrm{C}$ Oleate incorporation assay. $2.5 \times 10^{5} \mathrm{HeLa}$ cells were seeded in 6 -cm dishes, followed by transfection with mCherry-Vector, hAGPAT2-mCherry, hCDS1mCherry or hCDS2-mCherry. Cells were then treated with $1 \mu \mathrm{Ci}\left[{ }^{14} \mathrm{C}\right]$ oleic acid (PerkinElmer) conjugated to BSA in DMEM-10\%FBS medium for 30 min after reaching $~ 80 \%$ confluency. After washing cells twice with PBS, ${ }^{14} \mathrm{C}$-labelled TAG was extracted by a $2-\mathrm{mL}$ mixture of hexane (Ajax FineChem) and isopropanol (Ajax FineChem) (3:2) for $30 \mathrm{~min}$ in a fume hood. After transferring into 2-mL glass vials, another $1 \mathrm{~mL}$ of hexane:isopropanol (3:2) was used to collect the lipid residues and combined with the previous $2 \mathrm{~mL}$ solvent. Solvent was dried completely in the fume hood. Lipids were reconstituted in $60 \mu \mathrm{l}$ of hexane. The samples were then spotted on a Silica Gel 60 plate (Millipore) and separated in a solvent system consisting of heptane/diethyl ether/glacial acetic acid (90:30:1) (Ajax FineChem). The TLC plate, after drying completely, was exposed to a BAS-MS imaging sheet (Fujifilm, Tokyo, Japan) for 5-7 days in an enclosed cassette at room temperature. ${ }^{14} \mathrm{C}$-labelled TAG was then visualized by Typhoon FLA 9500 phosphor imager (GE Healthcare). After the dishes were dried completely, $1 \mathrm{~mL} 0.1 \mathrm{M}$ $\mathrm{NaOH}$ (Ajax FineChem) was added to extracted protein. The protein concentrations were determined by a bicinchoninic acid (Sigma) assay.

${ }^{13}$ C-FA tracing and lipid extraction. $2 \times 10^{5} \mathrm{HeLa}$ cells were seeded overnight in six-well plates, followed by transfection with siRNA against AGPAT2 or mCherrytagged AGPAT2 for $24 \mathrm{~h}$. The media was then replaced with DMEM media containing $\left[\mathrm{U}^{13} \mathrm{C}\right]$-oleate (Sigma) in $5 \%$ dialyzed FBS (Sigma) and cells were incubated for $0,4,8$ and $16 \mathrm{~h}$ ( $n=3$ per each time point). Dialyzed FBS was used to avoid confounders in the form of serum metabolites, thereby enriching the 'tracer' signal While depleted of lipids, commercial dialyzed FBS contains growth factors and other nutrients so that it does not lead to cell growth arrest. At each time point cells were harvested and lipid lipids extracted using protocol described below. Chloroform and butanol extraction were both carried out to cover broader range of lipid classes. For chloroform extraction, cells were washed twice with ice-cold PBS and quenched by the addition of $0.75 \mathrm{~mL}$ ice cold methanol/PBS (1:1) on ice. The cells were then stored in $-20^{\circ} \mathrm{C}$ for $15 \mathrm{~min}$. Thereafter, cells were scraped and transferred into pre-chilled $1.5 \mathrm{~mL}$ centrifuge tube, mixed with $50 \mu \mathrm{L} 1 \mathrm{mM}$ 3,5-di-tert4-butylhydroxytoluene (BHT, Sigma), $1 \mu \mathrm{L}$ SPLASH Lipidomix internal standard mix (Avanti Polar Lipids) per $1 \times 10^{5}$ cells, $0.5 \mathrm{~mL}$ chloroform (Sigma). The mixture was vortexed vigorously for 1 min followed by centrifugation at $12,000 \times g$ for $5 \mathrm{~min}$. The lower chloroform layer was then collected into a fresh amber LC glass autosampler vial and dried under nitrogen gas. Samples were reconstituted at a concentration $20 \mu \mathrm{L}$ per $1 \mathrm{mg}$ of protein in chloroform/methanol $(1: 1, \mathrm{v} / \mathrm{v})$ prior to the LC-MS analysis. For butanol extraction, cells were washed twice with icecold PBS. The cells were placed on ice and $0.6 \mathrm{~mL}$ ice-cold buffer containing $15 \mathrm{mM}$ citric acid and $20 \mathrm{mM}$ disodium hydrogen phosphate ( $\mathrm{pH} 4.0)$ was added to the cells. The cells were then scraped into ice cold $2 \mathrm{~mL}$ centrifuge tube, mixed with $50 \mu \mathrm{L} 1 \mathrm{mM}$ BHT, $1 \mu \mathrm{L}$ SPLASH Lipidomix internal standard mix per $2 \times 10^{5}$ cells and $1 \mathrm{~mL}$ 1-butanol (Sigma). The mixture was vortexed vigorously for $10 \mathrm{~min}$ followed by centrifugation at $12,000 \times g$ for $5 \mathrm{~min}$. The upper butanol layer was then collected into a fresh amber LC glass autosampler vial, and butanol extraction was repeated by the addition of $0.5 \mathrm{~mL}$ butanol. The butanol fractions were combined and dried under nitrogen gas. Samples were reconstituted in $100 \mu \mathrm{L}$ methanol (Sigma) prior to the LC-MS analysis.

Liquid chromatography-mass spectrometry (LC-MS) analyses. Lipidomic analyses were performed using an Agilent 6560 ion mobility Q-TOF LC-MS coupled to a 1290 Infinity II UHPLC system. Chromatographic conditions were 
used as described previously ${ }^{43}$. For chloroform and butanol extracted lipids, $5 \mu \mathrm{L}$ of sample was injected onto $1.7 \mu \mathrm{m}$ particle $100 \times 2.1 \mathrm{~mm}$ ID Waters Acquity CSH C18 column. Chloroform extracted lipids were separated using a gradient of mobile phase A (water/acetonitrile, 4:6, v/v) with $10 \mathrm{mM}$ ammonium formate and mobile phase B (acetonitrile/2-propanol, 1:9, v/v) with $10 \mathrm{mM}$ ammonium formate at a flow rate of $0.3 \mathrm{~mL} / \mathrm{min}$ and column temperature maintained at $55^{\circ} \mathrm{C}$. The gradient ran from $0 \%$ to $40 \%$ mobile phase B from 0 to 6 min, increased from $40 \%$ to $100 \%$ mobile phase B over the next $24 \mathrm{~min}$, maintained at $100 \%$ mobile phase B for another $4 \mathrm{~min}$, and then returned to $0 \%$ mobile phase B over 2 min followed by $4 \mathrm{~min}$ to condition the column ( $40 \mathrm{~min}$ total). Lipids were analysed in positive ionization polarity mode. The electrospray settings were as follows: gas temperature $30{ }^{\circ} \mathrm{C}$; drying gas flow $5 \mathrm{~L} / \mathrm{min}$; sheath gas temperature $300^{\circ} \mathrm{C}$; sheath gas flow $12 \mathrm{~L} / \mathrm{min}$; cap voltage $3.5 \mathrm{kV}$. For 'unlabelled' $T=0 \mathrm{~h}$ sample, data acquisition was performed using an Auto MS/MS mode, and for ${ }^{13} \mathrm{C}$-labelled samples data acquisition was performed using a full scan $\mathrm{MS}^{1}$ mode. The scan range was between 100 and $1700 \mathrm{~m} / z$. For Auto MS mode collision energy was $35 \mathrm{eV}, 2$ precursors per cycle and active exclusion for 0.5 min after 2 spectra. Mass correction was done by constant infusion of 'lock mass' ions $(\mathrm{m} / \mathrm{z} 121.0509$ and $\mathrm{m} / \mathrm{z}$ 922.0098).

Butanol extracted lipids were separated using a gradient of mobile phase A $\left(0.05 \% \mathrm{NH}_{4} \mathrm{OH}\right.$ in water) and mobile phase $\mathrm{B}\left(0.05 \% \mathrm{NH}_{4} \mathrm{OH}\right.$ in methanol) at a flow rate $0.2 \mathrm{~mL} / \mathrm{min}$ and column temperature maintained at $50^{\circ} \mathrm{C}$. The gradient elution programme was as follows: $0-1 \mathrm{~min}$ isocratic $55 \% \mathrm{~B}, 1-14 \mathrm{~min} 55-85 \% \mathrm{~B}$, 14-20 min $85-100 \%$ B, $20-22$ min $100 \%$ B, 22-26 min return to $55 \%$ B followed by column equilibration for $4 \mathrm{~min}(30 \mathrm{~min}$ total). Lipids were analysed in negative ionization polarity mode with the same MS source temperature and voltage parameters as above. The scan range was between 100 and $1100 \mathrm{~m} / z$. Mass correction was done by constant infusion of 'lock mass' ions $(\mathrm{m} / \mathrm{z} 112.9856$ and $\mathrm{m} / \mathrm{z}$ 1033.9881)

Lipidomic data processing and analysis. Peak detection and lipid identification was performed using Agilent Lipid Annotator 1.0. The list of identified lipids was converted into.csv file and used by MAVEN software ${ }^{44}$ as a library to analyse data acquired from stable isotope tracing experiment. Unlabelled and labelled intensities for each lipid were extracted, an unlabelled pool $\left(X^{\mathrm{U}}\right)$ of each lipid class was calculated by summing up the intensities of unlabelled $(M+0)$ lipids, a labelled pool $\left(X^{\mathrm{L}}\right)$ of each lipid class was calculated by summing up the intensities of ${ }^{13} \mathrm{C}$ oleate-containing lipids $(M+18, M+36$ and $M+54)$. Fluxes through individual lipid classes were calculated using kinetic flux profiling approach ${ }^{45}$, assuming cells were under metabolic steady state.

Analyses of liver PA. Lipids were extracted from mouse liver slices following a modified Bligh and Dyer's protocol as previously described ${ }^{46}$. The obtained lipidome was quantified using a targeted lipidomic approach ${ }^{46}$. Analyses of PA was conducted on a system comprising an Exion-UPLC coupled with a 6500 Plus QTRAP mass spectrometer (Sciex).

Neutral lipid extraction. HeLa cells were grown in 10-cm dishes. $400 \mu \mathrm{M}$ oleate was added to cells for $18 \mathrm{~h}$ when cells reached $80-90 \%$ confluence. A Triglyceride Assay Kit (Abcam) was used to determine the TAG levels according to the manufacturer's protocol.

Generation of the A2LKO mice. The Ethics Committee at Model Animal Research Center of Nanjing University approved all animal procedures used in this study, which comply with all relevant ethical regulations. Mice were housed under a light/ dark cycle of $12 \mathrm{~h}$ with free access to food and water unless otherwise stated. Liverspecific AGPAT2 knock out mice in C57Bl/6J background were generated by the transgenic facility at Nanjing University in collaboration with GemPharmatech Co. The CRISPR/Cas9-based strategy used to disrupt Agpat2 in mice is outlined in Fig. S7A: two Lox $P$ sites were introduced into the Agpat2 locus, sandwiching exons 2 and 4 . Briefly, sgRNA was transcribed in vitro and donor vector was constructed accordingly. Cas9, sgRNA and donor were microinjected into the fertilized eggs of C57BL/6J mice. Fertilized eggs were then transplanted to obtain positive F0 mice which were confirmed by PCR and sequencing. A stable F1 generation mouse model was obtained by mating positive F0 generation mice with C57BL/6J mice. Homozygous Agpat $2^{\mathrm{f} / / \mathrm{fl}}$ mice were then crossed with transgenic mice expressing Cre recombinase under the control of the albumin promoter. The resulting Agpat $2^{\mathrm{tl} /}$ ${ }^{+} \mathrm{Alb}^{-c r e T g / 0}$ progeny was then crossed with Agpat $2^{\mathrm{fl} / \mathrm{fl}}$ mice to generate the A2LKO mice. Diet-induced obesity studies were carried out by feeding mice a HFD $(60 \%$ calories from fat, Research Diets D12492) for 15 weeks at the age of 8 weeks.

Triacylglycerol and cholesterol levels in liver. For Liver TAG levels, frozen liver chunks were saponified in ethanolic $\mathrm{KOH}$, and the extracts were subsequently neutralized. The resultant free glycerol was determined with the Free Glycerol Reagent (F6428, Sigma-Aldrich) using glycerol (Sigma-Aldrich) as standard for calculation. For liver TC levels, lipids were extracted with chloroform:isopropanol:NP-40
(7:11:0.1), and TC was measured after removal of organic solvent using LabAssay Cholesterol kit (Wako Chemicals, USA).

Primary mouse hepatocytes isolation and cell transfection. Primary mouse hepatocytes were isolated from 3-month-old mice using a collagenase-based method and cultured as previously described ${ }^{47}$. Hepatocytes from preparations with a cell viability $\geq 90 \%$ were seeded in six-well plates at a density of $1 \times 10^{6}$ per well in a plating medium (M199 with GlutaMAXTM supplemented with $100 \mathrm{U} / \mathrm{mL}$ penicillin, $0.1 \mathrm{mg} / \mathrm{mL}$ streptomycin, $0.1 \%$ bovine serum albumin (BSA), $10 \%(\mathrm{v} / \mathrm{v})$ foetal bovine serum, $10 \mathrm{nM}$ insulin, $200 \mathrm{nM}$ triiodothyronine, $500 \mathrm{nM}$ dexamethasone) for $4 \mathrm{~h}$ and then incubated overnight $(16 \mathrm{~h})$ in a recovery medium (M199 with GlutaMAXTM supplemented with $100 \mathrm{U} / \mathrm{mL}$ penicillin, $0.1 \mathrm{mg} / \mathrm{mL}$ streptomycin and $10 \%(\mathrm{v} / \mathrm{v})$ foetal bovine serum). The next morning, cells were used for transfection by plasmids with Lipofectamine-3000 (Thermo Fisher Scientific).

Immunofluorescence staining and imaging. Primary hepatocytes were fixed by $4 \%$ paraformaldehyde, followed by permeabilization with $0.1 \%$ Triton X-100 in PBS for $10 \mathrm{~min}$. The cells and the frozen tissue sections were stained by DAPI and BODIPY for $30 \mathrm{~min}$. Coverslips were then mounted onto slides by using $50 \%$ glycerol in PBS. All images were obtained by Olympus FV1000 laser scanning confocal microscope (Olympus, Tokyo, Japan) with $\times 60$ Plan Apochromat (1.4 NA) oil objective. Acquired images were quantified by ImageJ software.

Primer information for qPCR analysis of expression of target genes. All primers and protocols used for expression analyses of selected mouse liver genes were from a previous study ${ }^{11}$, except CDS1 and CDS2 (Supplementary Table 3).

CDS activity assay using mouse liver. CDS activity assay were carried out as described above except $100 \mu \mathrm{g}$ liver tissues were used and the reaction was incubated at $30^{\circ} \mathrm{C}$ for $30 \mathrm{~min}$.

Statistics and reproducibility. Data were analysed via $t$-test for two groups, or via one-way or two-way ANOVA for multiple groups using the Prism software (GraphPad, San Diego, CA, USA). Differences were considered statistically significant at $p<0.05$.

Reporting summary. Further information on research design is available in the Nature Research Reporting Summary linked to this article.

\section{Data availability}

A reporting summary for this article is available as Supplementary Information file. The main data supporting the findings of this study are available within the article and its Supplementary Figures. The exact $P$ values for the data are also included within the Source Data file. Additional details on datasets and protocols that support the findings of this study will be made available by the corresponding author upon reasonable request. Source data are provided with this paper.

Received: 1 October 2021; Accepted: 10 November 2021; Published online: 25 November 2021

\section{References}

1. Olzmann, J. A. \& Carvalho, P. Dynamics and functions of lipid droplets. Nat. Rev. Mol. Cell Biol. 20, 137-155 (2019).

2. Farese, R. V. Jr. \& Walther, T. C. Lipid droplets finally get a little R-E-S-P-EC-T. Cell 139, 855-860 (2009)

3. Yang, H., Galea, A., Sytnyk, V. \& Crossley, M. Controlling the size of lipid droplets: lipid and protein factors. Curr. Opin. Cell Biol. 24, 509-516 (2012).

4. Rosen, E. D. \& Spiegelman, B. M. What we talk about when we talk about fat Cell 156, 20-44 (2014).

5. Fei, W., Du, X. \& Yang, H. Seipin, adipogenesis and lipid droplets. Trends Endocrinol. Metab. 22, 204-210 (2011).

6. Agarwal, A. K. Lysophospholipid acyltransferases: 1-acylglycerol-3-phosphate O-acyltransferases. From discovery to disease. Curr. Opin. Lipidol. 23, 290-302 (2012)

7. Bradley, R. M. \& Duncan, R. E. The lysophosphatidic acid acyltransferases (acylglycerophosphate acyltransferases) family: one reaction, five enzymes, many roles. Curr. Opin. Lipidol. 29, 110-115 (2018).

8. Blunsom, N. J. \& Cockcroft, S. CDP-Diacylglycerol synthases (CDS): gateway to phosphatidylinositol and cardiolipin synthesis. Front. Cell Dev. Biol. 8, 63 (2020) 
9. Agarwal, A. K. et al. AGPAT2 is mutated in congenital generalized lipodystrophy linked to chromosome 9q34. Nat. Genet. 31, 21-23 (2002).

10. Agarwal, A. K. et al. Human 1-acylglycerol-3-phosphate O-acyltransferase isoforms 1 and 2: biochemical characterization and inability to rescue hepatic steatosis in Agpat2(-/-) gene lipodystrophic mice. J. Biol. Chem. 286, 37676-37691 (2011).

11. Cortes, V. A. et al. Molecular mechanisms of hepatic steatosis and insulin resistance in the AGPAT2-deficient mouse model of congenital generalized lipodystrophy. Cell Metab. 9, 165-176 (2009).

12. Cautivo, K. M. et al. AGPAT2 is essential for postnatal development and maintenance of white and brown adipose tissue. Mol. Metab. 5, 491-505 (2016).

13. Gale, S. E. et al. A regulatory role for 1-acylglycerol-3-phosphate-O-acyltransferase 2 in adipocyte differentiation. J. Biol. Chem. 281, 11082-11089 (2006).

14. Fei, W. et al. Fld1p, a functional homologue of human seipin, regulates the size of lipid droplets in yeast. J. Cell Biol. 180, 473-482 (2008).

15. Fei, W. et al. A role for phosphatidic acid in the formation of "supersized" lipid droplets. PLoS Genet. 7, e1002201 (2011).

16. Pagac, M. et al. SEIPIN regulates lipid droplet expansion and adipocyte development by modulating the activity of glycerol-3-phosphate acyltransferase. Cell Rep. 17, 1546-1559 (2016).

17. Yan, R. et al. Human SEIPIN binds anionic phospholipids. Dev. Cell 47, 248-256 e244 (2018).

18. Soltysik, K. et al. Nuclear lipid droplets form in the inner nuclear membrane in a seipin-independent manner. J. Cell Biol, 220, https://doi.org/10.1083/ jcb.202005026 (2021).

19. Tsukahara, T. et al. Phospholipase D2-dependent inhibition of the nuclear hormone receptor PPARgamma by cyclic phosphatidic acid. Mol. Cell 39, 421-432 (2010).

20. Stapleton, C. M. et al. Lysophosphatidic acid activates peroxisome proliferator activated receptor-gamma in $\mathrm{CHO}$ cells that over-express glycerol 3-phosphate acyltransferase-1. PLoS ONE 6, e18932 (2011).

21. Shin, J. J. \& Loewen, C. J. Putting the $\mathrm{pH}$ into phosphatidic acid signaling. BMC Biol. 9, 85 (2011).

22. Gao, M., Huang, X., Song, B. L. \& Yang, H. The biogenesis of lipid droplets: lipids take center stage. Prog. Lipid Res. 75, 100989 (2019).

23. Chung, J. et al. LDAF1 and seipin form a lipid droplet assembly complex. Dev. Cell 51, 551-563 e557 (2019).

24. Szymanski, K. M. et al. The lipodystrophy protein seipin is found at endoplasmic reticulum lipid droplet junctions and is important for droplet morphology. Proc. Natl Acad. Sci. USA 104, 20890-20895 (2007).

25. Yamashita, A. et al. Glycerophosphate/acylglycerophosphate acyltransferases. Biology (Basel) 3, 801-830 (2014).

26. Walther, T. C., Chung, J. \& Farese, R. V. Jr Lipid Droplet Biogenesis. Annu. Rev. Cell Dev. Biol. 33, 491-510 (2017).

27. Kassan, A. et al. Acyl-CoA synthetase 3 promotes lipid droplet biogenesis in ER microdomains. J. Cell Biol. 203, 985-1001 (2013)

28. Ben M'barek, K. et al. ER membrane phospholipids and surface tension control cellular lipid droplet formation. Dev. Cell 41, 591-604 e597 (2017).

29. Wang, $H$. et al. Seipin is required for converting nascent to mature lipid droplets. eLife 5, https://doi.org/10.7554/eLife.16582 (2016).

30. $\mathrm{Xu}, \mathrm{Y}$. et al. CDP-DAG synthase 1 and 2 regulate lipid droplet growth through distinct mechanisms. J. Biol. Chem. 294, 16740-16755 (2019).

31. Kassas, N. et al. Comparative characterization of phosphatidic acid sensors and their localization during frustrated phagocytosis. J. Biol. Chem. 292, 4266-4279 (2017).

32. Qi, Y. et al. CDP-diacylglycerol synthases regulate the growth of lipid droplets and adipocyte development. J. Lipid Res. 57, 767-780 (2016).

33. Srere, P. A. Complexes of sequential metabolic enzymes. Annu. Rev. Biochem. 56, 89-124 (1987).

34. Lee, J. \& Ridgway, N. D. Substrate channeling in the glycerol-3-phosphate pathway regulates the synthesis, storage and secretion of glycerolipids. Biochim. Biophys. Acta Mol. Cell Biol. Lipids 1865, 158438 (2020).

35. Reue, K. \& Dwyer, J. R. Lipin proteins and metabolic homeostasis. J. Lipid Res. 50(Suppl.), S109-S114 (2009).

36. Han, G. S., Wu, W. I. \& Carman, G. M. The Saccharomyces cerevisiae Lipin homolog is a $\mathrm{Mg}^{2+}$-dependent phosphatidate phosphatase enzyme. J. Biol. Chem. 281, 9210-9218 (2006).

37. Sankella, S., Garg, A., Horton, J. D. \& Agarwal, A. K. Hepatic gluconeogenesis is enhanced by phosphatidic acid which remains uninhibited by insulin in lipodystrophic Agpat2-/- mice. J. Biol. Chem. 289, 4762-4777 (2014).

38. Salo, V. T. et al. Seipin regulates ER-lipid droplet contacts and cargo delivery. EMBO J. 35, 2699-2716 (2016)
39. Du, X. \& Yang, H. Seipin regulates the formation of nuclear lipid droplets from a distance. J. Cell Biol. 220, https://doi.org/10.1083/jcb.202011166 (2021).

40. Ran, F. A. et al. Genome engineering using the CRISPR-Cas9 system. Nat. Protoc. 8, 2281-2308 (2013).

41. Fueller, J. et al. CRISPR-Cas12a-assisted PCR tagging of mammalian genes. J. Cell Biol. 219, https://doi.org/10.1083/jcb.201910210 (2020).

42. Blunsom, N. J., Gomez-Espinosa, E., Ashlin, T. G. \& Cockcroft, S. Mitochondrial CDP-diacylglycerol synthase activity is due to the peripheral protein, TAMM41 and not due to the integral membrane protein, CDPdiacylglycerol synthase 1. Biochim. Biophys. Acta Mol. Cell Biol. Lipids 1863, 284-298 (2018).

43. Ackerman, D. et al. Triglycerides promote lipid homeostasis during hypoxic stress by balancing fatty acid saturation. Cell Rep. 24, 2596-2605 e2595 (2018)

44. Melamud, E., Vastag, L. \& Rabinowitz, J. D. Metabolomic analysis and visualization engine for LC-MS data. Anal. Chem. 82, 9818-9826 (2010).

45. Yuan, J., Bennett, B. D. \& Rabinowitz, J. D. Kinetic flux profiling for quantitation of cellular metabolic fluxes. Nat. Protoc. 3, 1328-1340 (2008).

46. Lam, S. M. et al. A multi-omics investigation of the composition and function of extracellular vesicles along the temporal trajectory of COVID-19. Nat. Metab. 3, 909-922 (2021).

47. Patel, K. et al. The LKB1-salt-inducible kinase pathway functions as a key gluconeogenic suppressor in the liver. Nat. Commun. 5, 4535 (2014).

\section{Acknowledgements}

H.Y. is supported by grants $(1078117,1141938,1141939$ and 1144726$)$ from the National Health and Medical Research Council (NHMRC), Australia. S.C. is supported by Grant No. 2018YFA0801102 from the Ministry of Science and Technology of China.

\section{Author contributions}

H.Y.M., Q.O., S.T., J.X., F.D., P.R., X.W., I.L., S.M.L. and X.D. performed experiments, analysed data, and contributed to the writing of the final submitted version of the manuscript. M.G., A.J.B., G.S., X.G., R.S., X.H. and S.C. analysed data and guided research. H.Y. designed research, analysed data, and wrote the manuscript with all other authors. H.Y. is the guarantor of this work, has full access to all the data in the study, and takes responsibility for the integrity of the data and the accuracy of the data analysis.

\section{Competing interests}

The authors declare no competing interests.

\section{Additional information}

Supplementary information The online version contains supplementary material available at https://doi.org/10.1038/s41467-021-27279-4.

Correspondence and requests for materials should be addressed to Hongyuan Yang.

Peer review information Nature Communications thanks the anonymous reviewer(s) for their contribution to the peer review of this work

Reprints and permission information is available at http://www.nature.com/reprints

Publisher's noteSpringer Nature remains neutral with regard to jurisdictional claims in published maps and institutional affiliations.

Open Access This article is licensed under a Creative Common Attribution 4.0 International License, which permits use, sharing, adaptation, distribution and reproduction in any medium or format, as long as you give appropriate credit to the original author(s) and the source, provide a link to the Creative Commons license, and indicate if changes were made. The images or other third party material in this article are included in the article's Creative Commons license, unless indicated otherwise in a credit line to the material. If material is not included in the article's Creative Commons license and your intended use is not permitted by statutory regulation or exceeds the permitted use, you will need to obtain permission directly from the copyright holder. To view a copy of this license, visit http://creativecommons.org/ licenses/by/4.0/

(c) The Author(s) 2021 